\title{
Loss of the sphingolipid desaturase DEGS1 causes hypomyelinating leukodystrophy
}

Devesh C. Pant,,$^{1,2}$ Imen Dorboz, ${ }^{3}$ Agatha Schluter, ${ }^{1,2}$ Stéphane Fourcade, ${ }^{1,2}$ Nathalie Launay, ${ }^{1,2}$ Javier Joya, ${ }^{1,2}$ Sergio Aguilera-Albesa, ${ }^{4}$ Maria Eugenia Yoldi, ${ }^{4}$ Carlos Casasnovas, ${ }^{1,2,5}$ Mary J. Willis, ${ }^{6}$ Montserrat Ruiz, ${ }^{1,2}$ Dorothée Ville, ${ }^{7}$ Gaetan Lesca, ${ }^{8}$ Karine Siquier-Pernet, ${ }^{9,10}$ Isabelle Desguerre, ${ }^{9,10}$ Huifang Yan, ${ }^{11,12}$ Jingmin Wang, ${ }^{11}$ Margit Burmeister, ${ }^{12,13}$ Lauren Brady, ${ }^{14}$ Mark Tarnopolsky, ${ }^{14}$ Carles Cornet, ${ }^{15}$ Davide Rubbini, ${ }^{15}$ Javier Terriente, ${ }^{15}$ Kiely N. James, ${ }^{16}$ Damir Musaev, ${ }^{16}$ Maha S. Zaki, ${ }^{17}$ Marc C. Patterson, ${ }^{18}$ Brendan C. Lanpher, ${ }^{19}$ Eric W. Klee,,${ }^{19,20}$ Filippo Pinto e Vairo, ${ }^{19,20}$ Elizabeth Wohler, ${ }^{21}$ Nara Lygia de M. Sobreira, ${ }^{22}$ Julie S. Cohen, ${ }^{23}$ Reza Maroofian, ${ }^{24}$ Hamid Galehdari, ${ }^{25}$ Neda Mazaheri, ${ }^{25,26}$ Cholamreza Shariati, ${ }^{26,27}$ Laurence Colleaux, ${ }^{9,10}$ Diana Rodriguez, ${ }^{28,29}$ Joseph G. Gleeson, ${ }^{16}$ Cristina Pujades, ${ }^{30}$ Ali Fatemi, ${ }^{23,31}$ Odile Boespflug-Tanguy, ${ }^{3,32}$ and Aurora Pujol ${ }^{1,2,33}$

'Neurometabolic Diseases Laboratory, Bellvitge Biomedical Research Institute (IDIBELL), 08908 L'Hospitalet de Llobregat, Barcelona, Catalonia, Spain. ${ }^{2}$ Center for Biomedical Research on Rare Diseases (CIBERER), ISCIII, Madrid, Spain. ${ }^{3}$ NSSERM UMR 1141, DHU PROTECT, Paris Diderot University, Sorbonne Paris Cité, Paris, France. ${ }^{4}$ Pediatric Neurology Unit, Department of Pediatrics, Navarra Health Service, Navarrabiomed, Pamplona, Spain. ${ }^{5}$ Neuromuscular Unit, Neurology Department, Hospital Universitari de Bellvitge, c/Feixa Llarga s/n, 08907 L'Hospitalet de Llobregat, Barcelona, Spain. ${ }^{6}$ Department of Pediatrics, Naval Medical Center San Diego, San Diego, California, USA. ${ }^{7}$ Department of Neuropediatrics, Lyon University Hospital, Lyon, France. ${ }^{8}$ Department of Medical Genetics, Lyon University Hospital and GENDEV team CNRS UMR 5292, INSERM U1028, CRNL, and University Claude Bernard Lyon 1, Lyon, France. ${ }^{9}$ Paris Descartes - Sorbonne Paris Cité University, Imagine Institute, Paris, France. 10evelopmental Brain Disorders Laboratory, INSERM UMR 1163, Paris, France. "Department of Pediatrics, Peking University First Hospital, Beijing, China. ${ }^{12}$ Molecular \& Behavioral Neuroscience Institute, and ${ }^{13}$ Departments of Computational Medicine \& Bioinformatics, Psychiatry and Human Cenetics, University of Michigan, Ann Arbor Michigan, USA. ${ }^{14}$ Department of Pediatrics (Neuromuscular and Neurometabolics), McMaster Children's Hospital, Hamilton, Ontario, Canada. ${ }^{15} Z e C l$ linics SL, PRBB, Barcelona, Spain. ${ }^{16}$ Laboratory for Pediatric Brain Disease, Department of Neurosciences, Howard Hughes Medical Institute, University of California, San Diego, California, USA. ${ }^{17}$ Human Genetics and Genome Research Division, Clinical Genetics Department, National Research Centre, Cairo, Egypt. ${ }^{18}$ Departments of Neurology and Pediatrics, Mayo Clinic, Rochester, Minnesota, USA. ${ }^{19} \mathrm{Department}$ of Clinical Cenomics and ${ }^{20}$ Center for Individualized Medicine, Department of Health Sciences Research, Mayo Clinic, Rochester, Minnesota, USA. ${ }^{2}$ McKusick-Nathans Institute of Cenetic Medicine, Johns Hopkins University School of Medicine, Baltimore, Maryland, USA. ${ }^{22}$ McKusick-Nathans Institute of Genetic Medicine, and Department of Pediatrics, Johns Hopkins University School of Medicine, Baltimore, Maryland, USA. ${ }^{23}$ Moser Center for Leukodystrophies at the Kennedy Krieger Institute, Baltimore, Maryland, USA. ${ }^{24}$ Cenetics Research Centre, Molecular and Clinical Sciences Institute, St. Ceorge's, University of London, London, United Kingdom. ${ }^{25}$ Department of Genetics, Faculty of Science, Shahid Chamran University of Ahvaz, Ahvaz, Iran. ${ }^{26}$ Narges Medical Genetics and Prenatal Diagnosis Laboratory, Kianpars, Ahvaz, Iran. ${ }^{27}$ Department of Medical Genetics, Faculty of Medicine, Ahvaz Jundishapur University of Medical Sciences, Ahvaz, Iran. ${ }^{28} \mathrm{APHP}$, Department of Neuropediatrics, National Reference Center for Neurogenetic Disorders, Hôpital Armand-Trousseau, GHUEP, Paris, France. ${ }^{29} \mathrm{CRC}$ ConCer-LD, Sorbonne Universités, UPMC Université, Paris, France. ${ }^{30}$ Department of Experimental and Health Sciences, Universitat Pompeu Fabra, Barcelona, Spain. ${ }^{31}$ Department of Neurology, Johns Hopkins Medical Institutions, Baltimore, Maryland, USA. ${ }^{32}$ Assistance Publique des Hopitaux de Paris (APHP), Reference Center for Leukodystrophies and Rare Leukoencephalopathies (LEUKOFRANCE), Hôpital Robert Debré, Paris, France. ${ }^{33}$ Catalan Institution of Research and Advanced Studies (ICREA), Barcelona, Catalonia, Spain.

Sphingolipid imbalance is the culprit in a variety of neurological diseases, some affecting the myelin sheath. We have used whole-exome sequencing in patients with undetermined leukoencephalopathies to uncover the endoplasmic reticulum lipid desaturase DECS1 as the causative gene in 19 patients from 13 unrelated families. Shared features among the cases include severe motor arrest, early nystagmus, dystonia, spasticity, and profound failure to thrive. MRI showed hypomyelination, thinning of the corpus callosum, and progressive thalamic and cerebellar atrophy, suggesting a critical role of DECS1 in myelin development and maintenance. This enzyme converts dihydroceramide (DhCer) into ceramide (Cer) in the final step of the de novo biosynthesis pathway. We detected a marked increase of the substrate DhCer and DhCer/Cer ratios in patients' fibroblasts and muscle. Further, we used a knockdown approach for disease modeling in Danio rerio, followed by a preclinical test with the first-line treatment for multiple sclerosis, fingolimod (FTY720, Gilenya). The enzymatic inhibition of Cer synthase by fingolimod, 1 step prior to DECS1 in the pathway, reduced the critical DhCer/ Cer imbalance and the severe locomotor disability, increasing the number of myelinating oligodendrocytes in a zebrafish model. These proof-of-concept results pave the way to clinical translation.

Conflict of interest: The authors have declared that no conflict of interest exists. License: Copyright 2019, American Society for Clinical Investigation.

Submitted: August 16, 2018; Accepted: December 21, 2018.

Reference information: J Clin Invest. 2019;129(3):1240-1256.

https://doi.org/10.1172/JCI123959.

\section{Introduction}

Leukodystrophies (LDs) are a heterogeneous group of rare, heritable disorders of all ages that primarily affect the brain's white matter, often leading to motor and cognitive impairment in early childhood and progression to severe disability over time $(1,2)$. All 
modes of inheritance, broad pathogenic mechanisms, and complex cellular interactions underlie LDs, yet many cases remain unexplained with an unknown biochemical or molecular basis (3). With progress in clinical genomics, new forms of ultrarare LDs are being recognized (4-6), and advances in novel technologies in gene, cell, and enzyme therapy hold promise for improving patient management $(7,8)$.

Using whole-exome sequencing (WES), we have identified homozygous or compound heterozygous pathogenic variants in the DEGS1 gene in 19 LD patients from 13 unrelated families. DEGS1 was first cloned in 1996 from Drosophila melanogaster, and was given the name drosophila degenerative spermatocyte 1, or DEGS1 (9). It encodes a $\Delta 4$-dihydroceramide desaturase (OMIM 615843) mapping to chromosome 1q42.11, also known as DES1. This enzyme catalyzes the insertion of a $\Delta 4,5$-trans double bond into dihydroceramide (DhCer) to convert it to ceramide (Cer), in the final step of the de novo Cer biosynthesis pathway (Figure 1) (10).

Biosynthesis of Cer, made of a sphingoid base and a fatty acid, mainly occurs via 3 distinct pathways: (a) the de novo pathway, which takes place in the endoplasmic reticulum (ER) and uses palmitoyl-CoA and serine as its precursors; (b) the sphingomyelinase pathway, which takes place in the plasma membrane, Golgi apparatus, and mitochondria, and converts sphingomyelin into Cer bidirectionally; and (c) the salvage pathway, which converts complex sphingolipids species into Cer and recycles the acyl moiety of Cer in both lysosomes and endosomes (Figure 1) (11). This compartmentalization of the more than 200 structurally distinct Cer suggests a high complexity of regulation and function, which is only beginning to emerge $(12,13)$.

Cer is the central unit of all sphingolipids, serving as a building block and as a hub for bioactive, more complex lipidic species. The biosynthesis of Cer is followed by the addition of sugar moieties to generate glucosylceramide and galactosylceramide, which undergo further transformation into gangliosides and sulfatides, respectively. Galactosylceramides and sulfatides with very long $\mathrm{N}$-acyl chains, in particular C24:0 and C24:1, are the most abundant myelin lipid components $(14,15)$. Indeed, defects of galactosylceramidase and arylsulfatase A, 1 and 2 steps after DEGS1, respectively, cause Krabbe disease/globoid LD (GLD) and metachromatic LD (MLD) (Figure 1), 2 well-characterized demyelinating LDs. Beyond the myelin sheath, sphingolipids are involved in numerous biological processes related to cell survival, metabolic regulation, and adaptation to stressors (16). The importance of sphingolipids in neurodegenerative disease has recently expanded beyond lysosomal disorders or classical sphingolipidosis. Secondary disturbances of these lipid species including DhCer have been shown in Huntington disease (17) and Alzheimer's patients (18).

Evidence from studies in animal models supports an essential role of DEGS1 in development $(19,20)$. Homozygous Degs1 ${ }^{-/}$mice die within the first 8 weeks of age, presenting a complex phenotype, including small size, scaly skin, sparse hair, and tremors (19). Lipidomics analysis showed that Degs $1^{-/-}$mice exhibit accumulation of DhCer and higher DhCer/Cer ratios in several tissues (19), similarly to the D. melanogaster model (20).

Our patients presented with hypomyelinating LD with progressive atrophy of the corpus callosum (CC), thalami, and cere- bellum, severe failure to thrive, and peripheral neuropathy. Using patients' fibroblasts, we functionally validated variants by testing their impact on DhCer/Cer ratios and reactive oxygen species (ROS) production. Importantly, treatment with fingolimod (FTY720), a drug targeting sphingolipid metabolism and a broadly used treatment for relapsing-remitting multiple sclerosis (MS), improved the metabolic imbalance, numbers of myelin-producing oligodendrocytes, and locomotor deficits in a zebrafish model.

\section{Results}

Biallelic deleterious variants of DEGS1 in patients with brain white matter abnormalities. As part of our ongoing studies on the molecular basis underlying undiagnosed leukoencephalopathies, we identified a total of 19 individuals from 13 unrelated families with rare variants suspected to alter DEGS1 function (Figure 2 and Tables 1, 2, and 3). The first patient under investigation was a female who presented feeding difficulties since birth, extreme irritability, hypertonia with opisthotonus, and nystagmus, resulting in death at 18 months. Severe hypomyelination was observed in the central and peripheral nervous system in the MRI and nerve conduction studies (patient 6, Tables 1 and 4). Extensive diagnostic investigations were negative. We thus carried out WES (see Methods) in the proband, with subsequent Sanger validation and segregation analysis (Supplemental Figure 1; supplemental material available online with this article; https://doi. org/10.1172/JCI123959DS1). We identified a homozygous frameshift variant in DEGS1 (GenBank ID, NM_003676.3; c.604delT; p. [Tyr202Thrfs ${ }^{*}$ ]; http://www.ncbi.nlm.nih.gov/genbank/) that was not present in the genome aggregation database (GnomAD; >246,000 chromosomes; http://gnomad.broadinstitute.org/), the NHLBI Exome Variant Server (EVS; >13,000 alleles; http://evs. gs.washington.edu/EVS/), or the Exome Aggregation Consortium (ExAC) database (>60,706 individuals; http://exac.broadinstitute. org/). No homozygous loss-of-function (LoF) variants were present for this gene in these databases. Personal communication with international Reference Centers for Leukodystrophies and information exchange on the GeneMatcher (https://genematcher.org/) (21) platform facilitated the identification of 18 additional affected individuals with DEGS1 variants from various ethnic backgrounds who displayed overlapping phenotypes. These variants were present in a homozygous or compound heterozygous manner in 13 families and segregated in the recessive inheritance mode. Consanguinity was present in 2 out of 3 of the families. All consanguineous families harbor a homozygous variant except family 9.

DEGS1 encodes an evolutionarily conserved sphingolipid desaturase of 323 aa containing 6 transmembrane domains, 3 histidine motifs, a lipid desaturase domain, and a fatty acid desaturase (FAD) domain (Figure 2). The highly conserved histidine motifs $\mathrm{HX}_{(3-4)} \mathrm{H}, \mathrm{HX}_{(2-3)} \mathrm{HH}$, and $\mathrm{H} / \mathrm{QX}_{(2-3)} \mathrm{HH}$, are essential for catalytic activity (10). Twelve out of 13 variants are located in the FAD domain, and only one, p.(Met37Thr), is located in the sphingolipid $\triangle 4$-desaturase domain. The most prevalent DEGS1 variants in our cohort were p.(Asn255Ser) and p. $\left(\operatorname{Trp} 107^{*}\right)$, identified in 3 and 2 independent families, respectively. Eight out of 13 variants have not been identified in control populations, and the rest of the variants have a minor allele frequency (MAF) lower than 0.0001, with none being found in the homozygous state in the database 


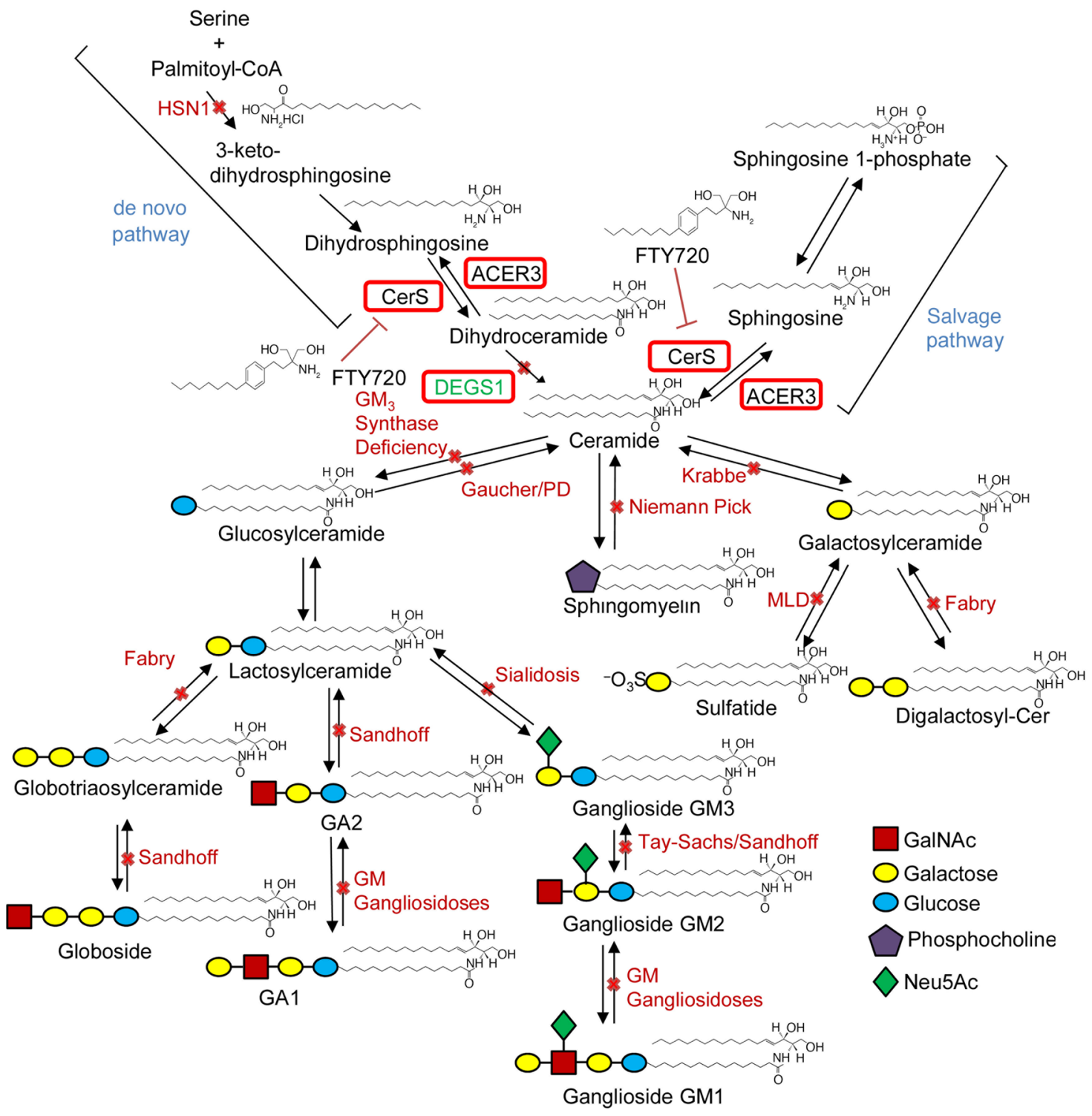

Figure 1. Scheme depicting enzyme defects associated with neurological disorders in the sphingolipid metabolism pathway, and fingolimod (FTY720) action. Serine palmitoyltransferase (SPT) catalyzes the initial reaction of the de novo sphingolipid pathway. Dihydrosphingosine is produced after an intermediate step regulated by 3-keto-dihydrosphingosine reductase (KDS), which is then followed by acylation by ceramide synthase (CerS) to produce dihydroceramide. The final reaction is the addition of a double bond by dihydroceramide desaturase (DECS1) to form ceramide. Ceramide is metabolized by ceramidase (CDse) to generate sphingosine, which in turn produces sphingosine 1-phosphate through phosphorylation by sphingosine kinase-1 and sphingosine kinase-2 (SphK1/2). Sphingosine 1-phosphate can be catabolized into hexadecenal and ethanolamine phosphate by sphingosine 1-phosphate lyase (S1PL). Ceramide can be generated by the breakdown of sphingomyelin (SM) by acid (ASM) or neutral sphingomyelinase (NSM). FTY720 has inhibitory effects on CerS. Enzyme (in bold) defects are indicated by solid bars across the blue arrows. The names of diseases are shown in red text. ACER3, alkaline ceramidase 3; GalCer, galactosylceramide; HSN1, hereditary sensory neuropathy type I; MLD, metachromatic leukodystrophy; PD, Parkinson disease; Sap, saposin.

of Genomic Variants (http://dgv.tcag.ca/dgv/app/home/) ExAC, GnomAD, 1000 Genomes (http://phase3browser.1000genomes. org/index.html), Iranome (http://www.iranome.ir/), or GME Variome (http://igm.ucsd.edu/gme/) database. Seven out of 13 variants are nonsense, with 4 stop-gain and 3 frameshift mutations. Six out of 13 variants are missenses with high deleteriousness predicted by most of the 15 in silico tools tested (Supplemental Table 1).

Clinical features. Among the 19 affected patients, the age of onset was 5.6 months $( \pm 7.2$; range $0.5-24$ months) with variable disease severity. Fifteen of the patients $(79 \%)$ presented a severe form characterized by a very poor psychomotor development, dys- tonia, and severe spasticity (Tables 1-3 and Supplemental Video). All except one subject presented early nystagmus or abnormal eye movements between 1 and 6 months of age. Seizures were frequently observed $(80 \%)$ around 2 years of age with a pharmacoresistant epilepsy sensitive to ketogenic diet in only 3 cases. In this group, failure to thrive was the most worrying clinical issue after 2 years of age despite feeding through gastrostomia. Head circumference was relatively conserved, with acquired microcephaly $(<2$ SD) in only 3 cases. Death occurred in 4 patients $(26 \%)$ at a mean age of 4.6 years $( \pm 1.9$; range $2.5-7$ years). These 4 patients harbored LoF variants (Tables 1-3). 
A



Patient 3

(p.Leu251Phefs*10)

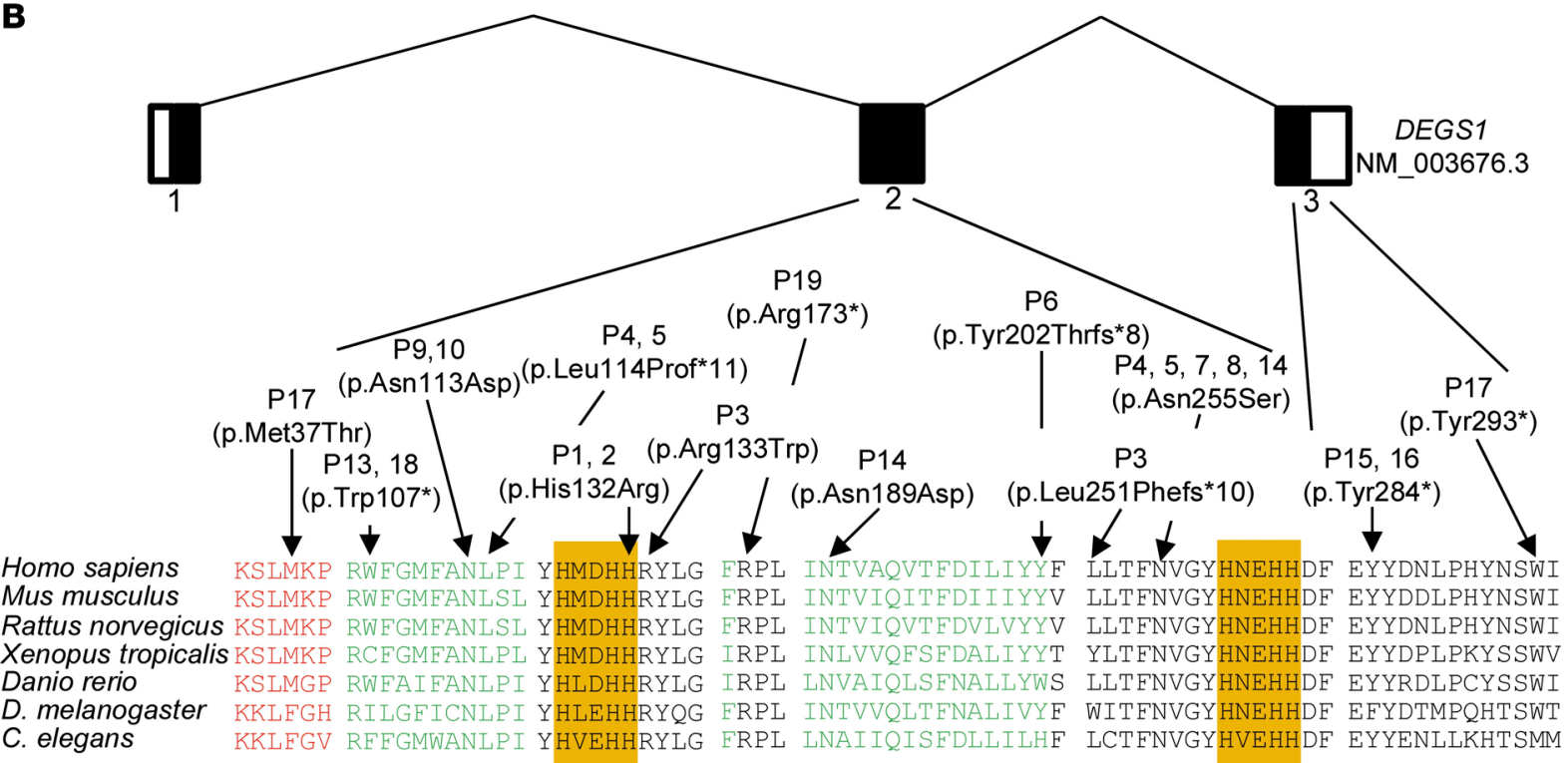

Figure 2. Schematic representation of human DEGS1 (NP_003667.1) and its functional domains with variants identified in patients. (A) DECS1 has 323 amino acids. Numbers on the scheme of the protein line indicate the boundaries of each transmembrane domain. The lipid DES domain (red) of DECS1 comprises amino acids 6-42. The histidine domains (orange) of DEGS1 comprise amino acids 89-93, 128-132, and 259-263. The transmembrane domains (green) of DECS1 comprise amino acids 43-62, 68-92, 104-122, 150-172, 184-202, and 210-231. The fatty acid desaturase (FAD) domain covers amino acids 64-293. (B) Schematic of the human DEGS1 locus, which consists of 3 exons. Patient ( $P$ ) numbers are indicated above the mutations. Multiple protein sequence alignment of DEGS1 orthologs show conservation of missense mutations detected in cases (bottom). The lipid DES, transmembrane, and histidine domains are indicated by red, green, and orange shading, respectively. The alignment was performed with ClustalW (http://www.clustal.org/) using the following RefSeq numbers: NP_003667.1, Homo sapiens; NP_031879.1, Mus musculus; NP_445775.2, Rattus norvegicus; NP_001007485.1, Xenopus tropicalis; NP_997865.1, Danio rerio; NP_476594.1, Drosophila melanogaster; NP_493549.1, Caenorhabditis elegans.

In the remaining $22 \%$ of the patients (patients $3,7,8$, and 14 ), a less severe phenotype was observed. Acquisition was better, with capacity to sit or walk and to use verbal communication. Progressive spasticity developed rapidly in these patients, leading to motor degradation at various ages ( 2 to 16 years). None presented with growth impairment (<2 SD) or microcephaly (Tables 1-3).

Cerebral MRI images were available for review by expert clinicians (AF, SAA, CC, DR, and OBT) for 18 patients at mean age 3.6 years ( \pm 4.2 ; range $0.5-17$ years) (Tables 4 and 5 ). All patients showed $\mathrm{T} 2$ and fluid-attenuated inversion recovery (T2/FLAIR) hyperintense white matter lesions, with a normal hyperintense $\mathrm{T} 1$ indicative of a hypomyelinating form of LD (Figure 3 and Supplemental Figure 2) (22). In the 3 mildest clinical forms of the disease (patients 7, 8, and 14), the abnormal white matter signal affected the deep white matter, with a relative preservation of the subcortical regions (Figure 3A, patient 7). In 2 severe cases a demyelinating aspect of the posterior subcortical-deep white matter was noticed on T1 (Supplemental Figure 2, patients 4 and 


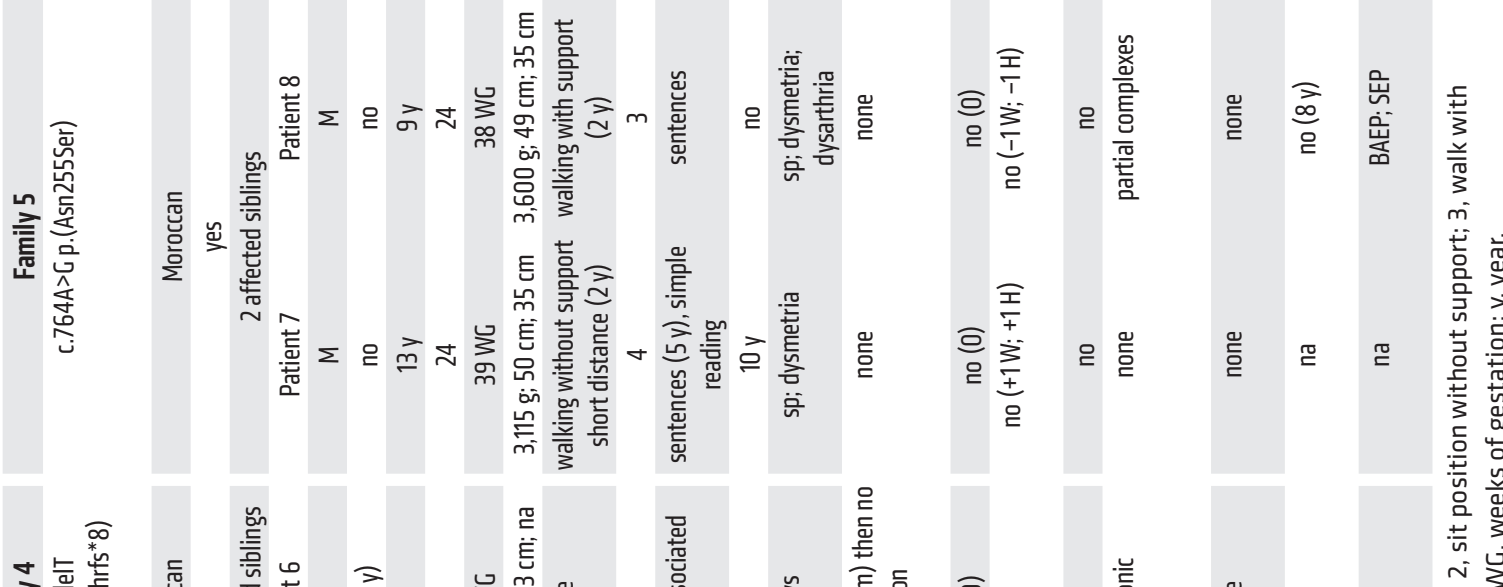

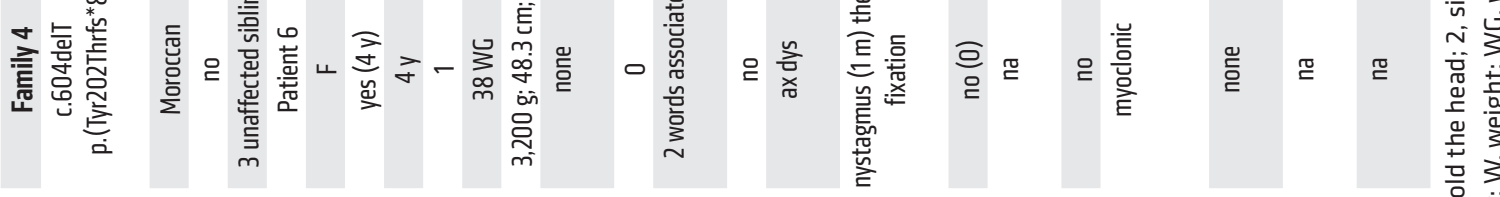

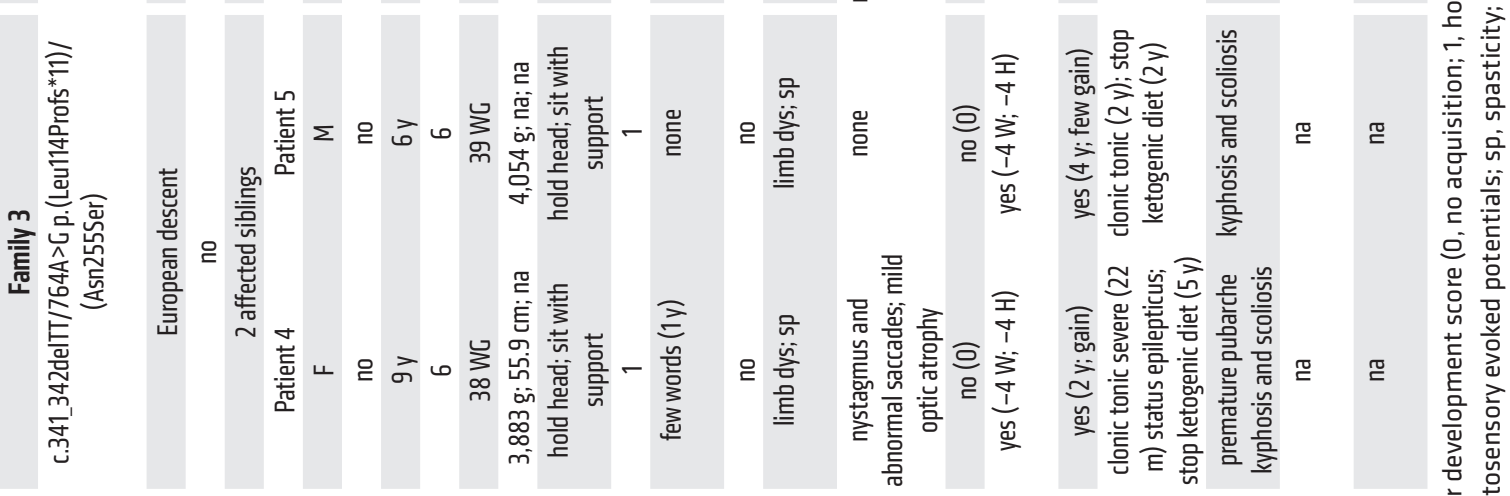

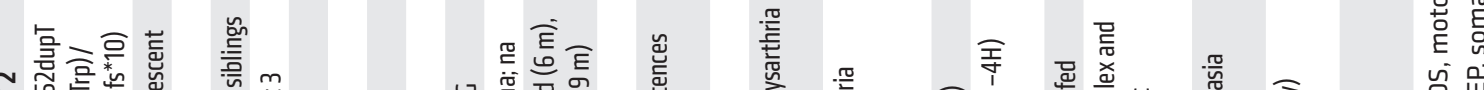

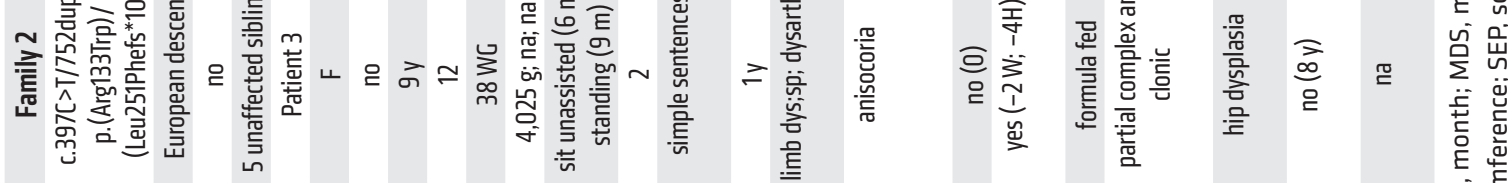

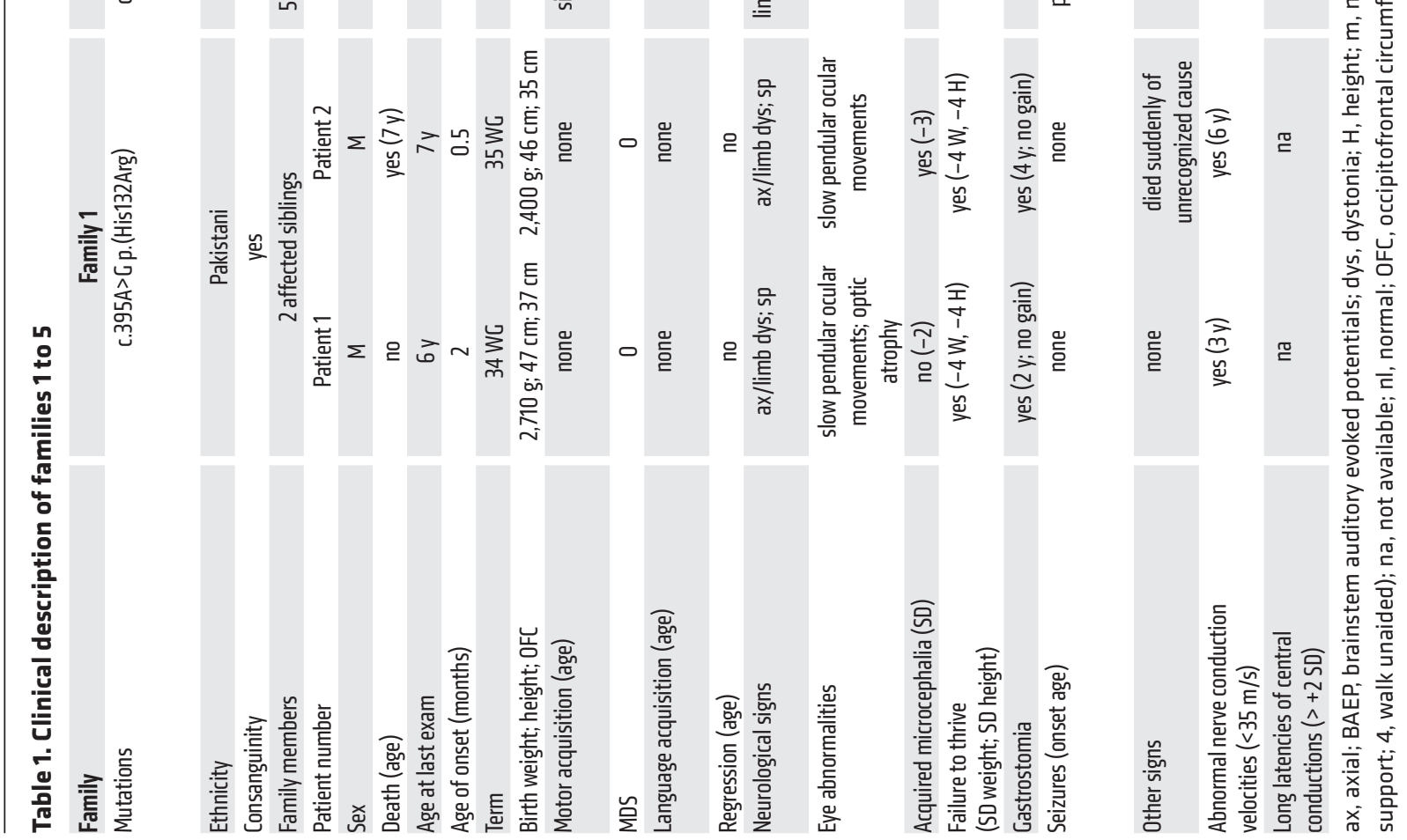




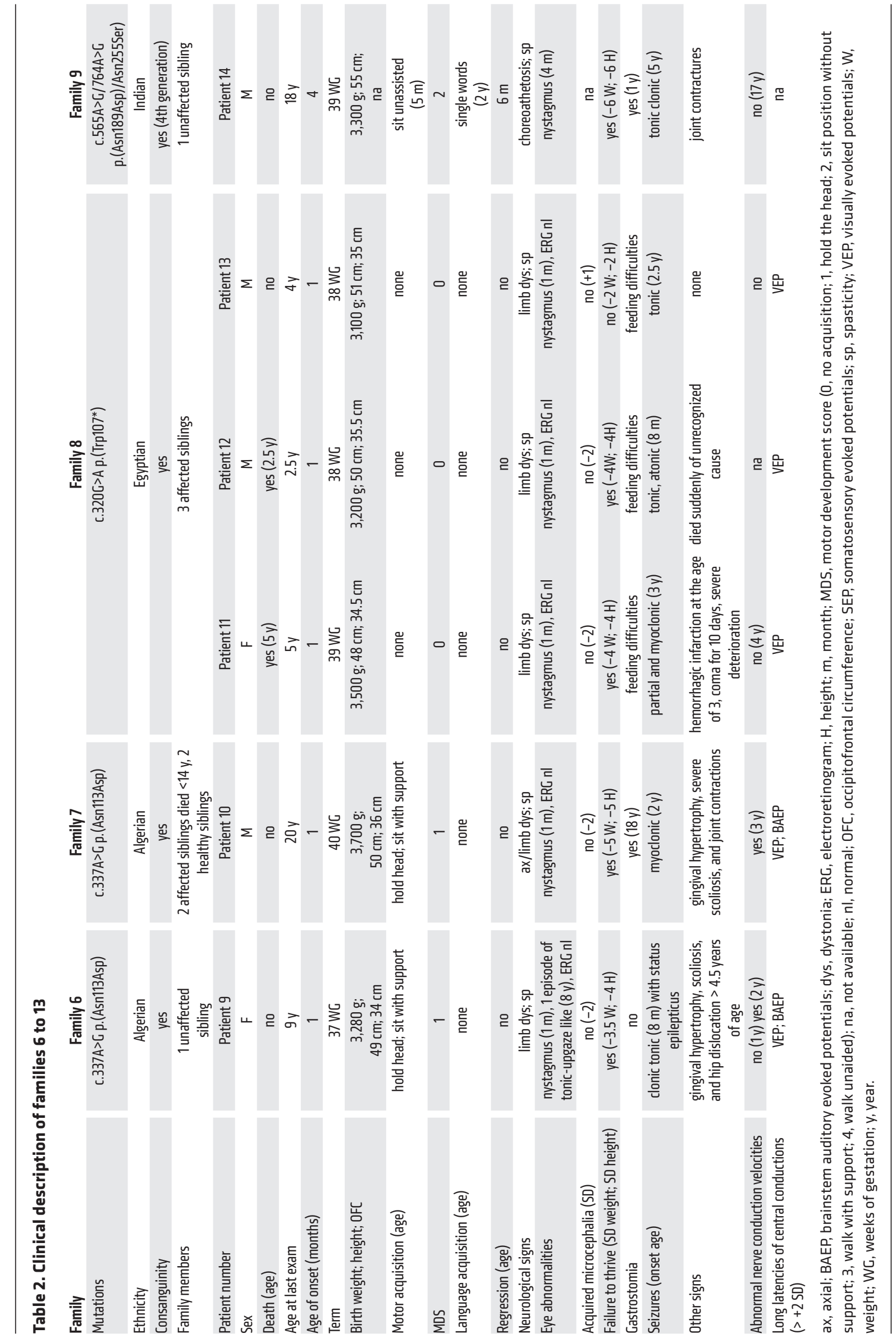


Table 3. Clinical description of families 10 to 13

\begin{tabular}{|c|c|c|c|c|c|}
\hline Family & \multicolumn{2}{|c|}{ Family 10} & Family 11 & Family 12 & Family13 \\
\hline Mutations & \multicolumn{2}{|c|}{ c.852_855del p.(Tyr284*) } & $\begin{array}{c}\text { c. } 110 T>C / 878 \mathrm{G}>\mathrm{A} \\
\text { p. }(\text { Met37Thr }) /\left(\operatorname{Trp} 293^{*}\right)\end{array}$ & c.320G>A p. $\left(\operatorname{Trp} 107^{*}\right)$ & c.517C>T p. $\left(\operatorname{Arg} 173^{*}\right)$ \\
\hline Ethnicity & \multicolumn{2}{|c|}{ European descent } & Chinese & Egyptian & Iranian-Lor \\
\hline Family members & \multicolumn{2}{|c|}{2 affected siblings } & 1 unaffected sibling & no sibling & no sibling \\
\hline Patient number & Patient 15 & Patient 16 & Patient 17 & Patient 18 & Patient 19 \\
\hline Sex & M & M & $\mathrm{F}$ & $\mathrm{F}$ & M \\
\hline Age at last exam & $1 y$ & $4 y$ & $5 y$ & $7.5 y$ & $10 y$ \\
\hline Age of onset (months) & 4 & 5 & 4 & 1 & 3 \\
\hline Term & 39 WG & $40 \mathrm{WG}$ & 39 WG & 38 WG & 39 WG \\
\hline Birth weight; height; OFC & 3,401 g; na; na & 3,515 g; na; na & 3,300 g; na; na & $2,500 \mathrm{~g} ; 48 \mathrm{~cm} ; 34 \mathrm{~cm}$ & $2,950 \mathrm{~g} ; 44 \mathrm{~cm} ; 34 \mathrm{~cm}$ \\
\hline Motor acquisition (age) & \multicolumn{2}{|c|}{ hold head; sit with support hold head; sit with support } & hold head & none & none \\
\hline MDS & 1 & 1 & 1 & 0 & 0 \\
\hline Eye abnormalities & nystagmus (4 m) & nystagmus (5 m) & none & nystagmus (1 m) & nystagmus \\
\hline Acquired microcephalia (SD) & no $(+1)$ & no $(+1)$ & na & yes $(-4.8)$ & yes $(-4)$ \\
\hline Failure to thrive (SD weight; SD height) & no $(-1 \mathrm{~W} ; 0 \mathrm{OH})$ & no $(-2 \mathrm{~W} ;-1 \mathrm{H})$ & no $(-1 \mathrm{~W} ;+2 \mathrm{H})$ & yes $(-3.5 \mathrm{~W} ;-6 \mathrm{H})$ & yes $(-4.5 \mathrm{~W} ;-3.5 \mathrm{H})$ \\
\hline Gastrostomia & yes $(1 \mathrm{y})$ & yes $(2.5 y)$ & no & no & $\begin{array}{l}\text { feeding difficulties, } \\
\text { dysphagia }\end{array}$ \\
\hline Seizures (onset age) & none & tonic clonic & none & febrile (4 y) & none \\
\hline Other signs & skin hypopigmentation & $\begin{array}{c}\text { scoliosis, skin } \\
\text { hypopigmentation }\end{array}$ & none & none & $\begin{array}{l}\text { kyphosis, coarse face, } \\
\text { hirsutism, bruxism }\end{array}$ \\
\hline Abnormal nerve conduction velocities & no (1y) & no $(4 y)$ & no $(4 y)$ & yes $(6 y)$ & na \\
\hline $\begin{array}{l}\text { Long latencies of central conductions } \\
(>+2 \text { SD) }\end{array}$ & BAEP & na & na & na & na \\
\hline
\end{tabular}

10). Despite the severity of the clinical symptoms, myelination progressed in the 6 patients analyzed with 2 or more MRI and a mean followup of 4.2 years $( \pm 3.5$; range $1-10$ years) (Figure $3 \mathrm{~B}$ and Supplemental Figure 2).

Basal ganglia abnormalities were observed in only the most severe forms. Abnormal hyperintense T2/FLAIR signal and atrophy of the thalami were the most frequent findings (11 of 15 cases, 73\%) (Figure 3, Tables 4 and 5, and Supplemental Figure 2). Two severe patients (patients 2 and 19) showed an associated hypointense T2/FLAIR in the pallidi (Tables 4 and 5).

Thinning of the CC was observed in $76 \%$ of cases associated, with progressive vermian cerebellar atrophy in $41 \%$ of cases (Figure 3 and Supplemental Figure 2). Demyelinating neuropathy with nerve conduction below $35 \mathrm{~m} / \mathrm{s}$ was observed in 5 out of the 14 patients analyzed by electromyography/nerve conduction velocity (1/3 of patients). The evoked potentials performed in 7 patients ( 4 brainstem auditory evoked potentials, 5 visually evoked potentials, and 1 somatosensory evoked potentials) demonstrated long latencies in the central conduction (>3 SD) in all cases (Tables 1-3).

Genotype-phenotype correlation. Patients from the same families (Tables 1-3; see families 1, 3, 5, 8, and 10) as well as patients with the same genotype (Tables 2 and 3, see Algerian families 6 and 7 and Egyptian families 8 and 12), expressed the same phenotype. Patients 2, 6, 11, and 12 who harbored homozygous LoF variants in the FAD domain, died at 7 years, 18 months, 2.5 years, and 5 years of age, respectively, indicating a likely phenotype-genotype correlation. At the other end of the spectrum we found patient 3, a 9-year-old girl with p.(Arg133Trp) and p.(Leu251Phefs*10) variants in trans, who presented with developmental regression from 9 months of age; however, she was able to sit without help and communicate using simple sentences. Her MRI and spectroscopy were almost normal at 4 and 5 years of age and presented no signs of peripheral neuropathy.

DEGS1 activity is impaired in patients' fibroblasts and muscle. To verify the impact of the variants on DEGS1 protein function, we used targeted lipidomics to quantify DhCer and Cer, the substrates and products of DEGS1, respectively. The results indicate a slight reduction of Cer concomitant with an important accumulation (3- to 9-fold) of DhCer in cultured skin fibroblasts of affected individuals (patients 4, 7, and 9) and muscle tissue (patient 3). Thus, the activity of the DEGS1 enzyme, represented as the DhCer/Cer ratio, was greatly decreased in all cases (Figure 4, A and B), consistent with the notion that the variants identified caused reduced activity of the DEGS1 enzyme. 


\section{Table 4. MRI descriptions patients 1-9}

\begin{tabular}{|c|c|c|c|c|c|c|c|c|c|}
\hline Patient number & 1 & 2 & 3 & 4 & 5 & 6 & 7 & 8 & 9 \\
\hline MRI (age of acquisition) & $5 \mathrm{~m}$ & $6 y$ & $8 y$ & $2 y ; 4 y$ & $2 y$ & $1 y$ & $6 y$ & $8 y$ & $8 \mathrm{~m} ; 2.5 \mathrm{y} ; 6 \mathrm{y}$ \\
\hline \multicolumn{10}{|c|}{ Hypomyelination according to age on the last MRI } \\
\hline cerebellum & + & no & no & no & no & + & no & no & + \\
\hline brainstem & no & no & no & no & no & no & no & no & + \\
\hline cC & + & + & no & no & no & no & no & no & no \\
\hline internal capsules & no & no & no & no & no & + & no & no & no \\
\hline periventricular WM & no & + & + & + & + & + & + & + & + \\
\hline deep WM & no & + & no & + & $+1-$ & + & no & no & + \\
\hline subcortical WM & no & $+1-$ & no & no & no & no & no & no & no \\
\hline myelin progression & na & na & na & yes & na & na & na & na & yes \\
\hline Basal ganglia abnormalities & $\begin{array}{l}\text { hyper T2 } \\
\text { thalami }\end{array}$ & $\begin{array}{c}\text { hypo T2 pallidi; } \\
\text { atrophy, } \\
\text { hyper T2 } \\
\text { thalami }\end{array}$ & no & $\begin{array}{l}\text { atrophy, } \\
\text { hyper T2 } \\
\text { thalami }\end{array}$ & $\begin{array}{l}\text { atrophy, } \\
\text { hyper T2 } \\
\text { thalami }\end{array}$ & no & no & no & $\begin{array}{l}\text { atrophy, } \\
\text { hyper T2 } \\
\text { thalami }\end{array}$ \\
\hline Other & none & $\begin{array}{l}\text { hypo T1 in the } \\
\text { occipital deep } \\
\text { WM/subcortical } \\
\text { junction }\end{array}$ & none & none & none & none & $\begin{array}{l}\text { enlargement } \\
\text { VR spaces }\end{array}$ & $\begin{array}{l}\text { enlargement } \\
\text { VR spaces; } \\
\text { spinal cord } \\
\text { normal }\end{array}$ & none \\
\hline Calcifications (CT scan) & no & $\begin{array}{c}\text { yes } \\
\text { (periventricular) }\end{array}$ & na & na & na & na & na & na & no \\
\hline \multicolumn{10}{|l|}{ Atrophy } \\
\hline cerebellum & no & ++ & no & no & no & no & no & no & + \\
\hline ventricle dilatation & + & + & no & no & no & + & no & no & no \\
\hline cortical & no & no & no & no & no & no & no & no & no \\
\hline$C C$ & no & ++ & no & + (splenium) & + (splenium) & + & no & no & + \\
\hline MRS & & & & & na & na & na & na & \\
\hline NAA & $\mathrm{nl}$ & low & $\mathrm{nl}$ & low & & & & & low \\
\hline choline & high & high & $\mathrm{nl}$ & high & & & & & $\mathrm{nl}$ \\
\hline lactates & - & + & - & - & & & & & $+1-$ \\
\hline
\end{tabular}

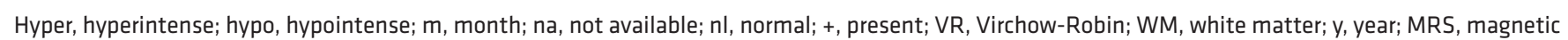
resonance spectroscopy; NAA, N-acetylaspartate.

Impaired function of DEGS1 induces intracellular ROS production. Excess of DhCer has been recently reported to generate ROS in $D$. melanogaster if - $\mathrm{KO}$ photoreceptors (20). We observed that ROS production was increased in all patients' fibroblasts (patients 4,7 , and 9) compared with controls (Figure 4C) using the probe 2',7'-dichlorodihydrofluorescein diacetate ( $\left.\mathrm{H}_{2} \mathrm{DCFDA}\right)$, which measures singlet oxygen, superoxide, hydroxyl radical, and various peroxides and hydroperoxides (23). To gain insight into the direct consequences of DhCer accumulation in our system, we incubated control and patients' fibroblasts with a long-chain DhCer (C18:0-DhCer) for 6 hours, at a dose of $20 \mu \mathrm{M}$. This dose increased ROS production in control fibroblasts, although we could not discern an increase in the patients' fibroblasts (Figure 4D). At higher doses, we found toxic effects in the fibroblasts independent of phenotype. This result confirmed that DhCer excess can generate ROS in human fibroblasts, whereas the blunted response in patient fibroblasts may follow saturation effects due to the steadystate accumulation of DhCer.

Degs1 is highly expressed in the central nervous system. We performed reverse transcription quantitative PCR (RT-qPCR) expression studies in different child and adult control human central nervous system (CNS) tissues $(n=2)$, to find specific expression of this gene in frontal lobe BA9, putamen, entorhinal cortex, hippocam- pus, hypothalamus, pons, cerebellum, white matter frontal cortex, and spinal cord (Supplemental Figure 3A). This is consistent with the expression of Degs1 mRNA in different tissues of 4-monthold wild-type mice $(n=3)$, with high expression in the CNS, in particular in the spinal cord, brain cortex, pons, cerebellum, and hippocampus, compared with other organs (Supplemental Figure 3B). We next performed RT-qPCR to assess the DEGS1 mRNA expression in fibroblasts from affected individuals (patients 4, 7, and 9) and healthy age-matched control individuals. We observed a 50\% decrease in patient 4 (Supplemental Figure 3C), possibly owing to mRNA decay in the allele bearing the stop variant. We also analyzed the expression of DEGS2, the closest paralog of DEGS1, which is reported to have a partially overlapping, low C4desaturase activity while showing high C4-hydroxylase activity $(24,25)$. We corroborated a high expression of DEGS2 in small intestine and kidney (24), comparable to DEGS1 (Supplemental Figure 3B), and 2 orders of magnitude lower expression in mouse brain and human fibroblasts as compared with DEGS1, which argues against any putative, physiologically relevant compensatory role of DEGS2 (Supplemental Figure 3, B and C).

Loss of Degs1 in zebrafish causes DhCer/Cer imbalance, reduced number of oligodendrocytes, and locomotor impairment. We investigated the suitability of a zebrafish model for this disease, since 


\section{Table 5. MRI descriptions patients 10-19}

\begin{tabular}{|c|c|c|c|c|c|c|c|c|c|c|}
\hline Patient number & 10 & 11 & 12 & 13 & 14 & 15 & 16 & 17 & 18 & 19 \\
\hline MRI (age of acquisition) & $2 \mathrm{y} ;$ & $2 y ;$ & $1 y$ & $10 \mathrm{~m}$ & $17 y$ & $11 \mathrm{~m}$ & $6 \mathrm{~m}$; & $8 \mathrm{~m}$; & $6 y$ & $10 y$ \\
\hline
\end{tabular}

\section{Hypomyelination according to age on the last MRI}

\begin{tabular}{|c|c|c|c|c|c|c|c|c|c|c|}
\hline cerebellum & + & no & + & + & no & no & no & no & na & no \\
\hline brainstem & no & no & + & no & no & no & no & no & na & no \\
\hline сC & no & no & no & + & no & $+1-$ & no & + & na & $+1-$ \\
\hline internal capsules & + & no & no & + & no & no & no & + & na & no \\
\hline periventricular WM & + & + & + & + & + & + & + & + & na & + \\
\hline deep WM & + & + & + & + & no & + & + & + & na & + \\
\hline subcortical WM & no & no & + & no & no & no & no & $+/-$ & na & no \\
\hline myelin progression & yes & yes & na & na & na & na & yes & yes & na & na \\
\hline Basal ganglia abnormalities & $\begin{array}{l}\text { atrophy, } \\
\text { hyper T2 } \\
\text { thalami }\end{array}$ & no & no & no & $\begin{array}{l}\text { atrophy, } \\
\text { hyper T2 } \\
\text { thalami }\end{array}$ & $\begin{array}{l}\text { hyper T2 } \\
\text { dentate } \\
\text { nucleus; } \\
\text { hyper T2 } \\
\text { thalami }\end{array}$ & $\begin{array}{c}\text { mild atrophy, } \\
\text { hyper FLAIR } \\
\text { thalami }\end{array}$ & $\begin{array}{l}\text { atrophy, } \\
\text { hyper T2 } \\
\text { thalami }\end{array}$ & na & $\begin{array}{l}\text { hypo T2 } \\
\text { pallidi; } \\
\text { atrophy, } \\
\text { hyper T2 } \\
\text { thalami }\end{array}$ \\
\hline Other & $\begin{array}{c}\text { hypo T1 in the } \\
\text { occipital deep } \\
\text { WM }\end{array}$ & $\begin{array}{c}\text { occipital } \\
\text { hemorrhagic } \\
\text { infarction at } 3\end{array}$ & none & none & none & none & no & no & na & no \\
\hline Calcifications (CT scan) & no & $\begin{array}{c}\text { yes } \\
\text { (cerebellum) }\end{array}$ & $\begin{array}{c}\text { yes } \\
\text { (cerebellum) }\end{array}$ & $\begin{array}{c}\text { yes } \\
\text { (cerebellum) }\end{array}$ & na & no & no & na & na & na \\
\hline \multicolumn{11}{|l|}{ Atrophy } \\
\hline cerebellum & ++ & + & + & no & + & no & + & no & na & ++ \\
\hline ventricle dilatation & + & + & no & no & no & no & + & no & na & + \\
\hline cortical & $\begin{array}{l}++ \text { (frontal } \\
\text { and Sylvian } \\
\text { fissures) }\end{array}$ & + & $\begin{array}{l}\text { + (Sylvian } \\
\text { fissures) }\end{array}$ & $\begin{array}{l}\text { + (Sylvian } \\
\text { fissures) }\end{array}$ & no & no & no & no & na & $\begin{array}{c}++ \text { (frontal } \\
\text { and Sylvian } \\
\text { fissures) }\end{array}$ \\
\hline CC & ++ & + & + & + & + & + & + & + (splenium) & na & ++ \\
\hline MRS & na & na & na & na & na & na & & na & na & na \\
\hline NAA & & & & & & & low & & & \\
\hline choline & & & & & & & $\mathrm{nl}$ & & & \\
\hline lactates & & & & & & & - & & & \\
\hline
\end{tabular}

Hyper, hyperintense; hypo, hypointense; m, month; na, not available; nl, normal; +, present; VR, Virchow-Robin; WM, white matter; y, year; MRS, magnetic resonance spectroscopy; NAA, N-acetylaspartate..

many of the genetic pathways for myelin development and maintenance are conserved (26). There is a single zebrafish ortholog of human DEGS1 (NP_997865.1), sharing 74\% protein identity with human DEGS1 (NP_003667.1). In situ hybridization (ISH) studies showed that Degs1 was expressed in the dorsal part of the zebrafish brain, being more prominent in the dorsal thalamus, posterior tuberculum, tectum opticum, hindbrain, and spinal cord (Supplemental Figure 4, A and B). Using a transgenic strain expressing EGFP under the control of the myelin basic protein $(\mathrm{mbp})$ promoter labeling myelinating oligodendrocytes $\left(\mathrm{MBP}^{+}\right)(27)$, we found an overlapping pattern between Degs1 and $\mathrm{MBP}^{+}$cells (Supplemental Figure 4, C-F). Thus, we knocked down Degs1 in zebrafish by designing an efficient splice-blocking morpholino (MO) (Supplemental Figure 4, G-I). Downregulation of DEGS1 induced an increase of DhCer and of the DhCer/Cer ratio (Figure $5 \mathrm{~A}$ ) at 5 days postfertilization ( $5 \mathrm{dpf}$ ). Moreover, a high percentage of MO-DEGS1 larvae displayed an abnormal morphology (Figure 5, B and C).

Next, phenotype and swimming ability of MO-DEGS1 zebrafish larvae were analyzed by measuring the total movement distance and by comparing the activity displayed by uninjected and MO-control-injected larvae. The total movement distance $(\mathrm{mm})$ in MO-DEGS1 larvae was very significantly reduced (Figure 5, D and E). Importantly, when embryos were injected with 10 ng of MO-DEGS1, no significant differences were observed in the survival rate compared to embryos injected with MOcontrols or uninjected animals. To test the effect of DEGS1 knockdown on myelination in zebrafish, we measured the number of $\mathrm{MBP}^{+}$cells in the spinal cord at $4.5 \mathrm{dpf}$ after MO-DEGS1 injection (27). Interestingly, we found that the number of $\mathrm{MBP}^{+}$ cells was reduced by $30 \%$ in MO-DEGS1 compared with control larvae (Figure 5, F, G, I, J, and M). Altogether, these results indicate that loss of DEGS1 function in zebrafish larvae results in a 10-fold increased DhCer/Cer ratio, locomotor disability, and impaired myelination, consistent with the patients' phenotype.

FTY720 ameliorates the phenotype of MO-DEGS1 zebrafish, while reducing ROS levels in patient fibroblasts. Drug screening in zebrafish has been successfully used to identify target pathways as well as therapeutic compounds for human diseases (28). 
$\mathbf{A}$


Patient 7 (6y)

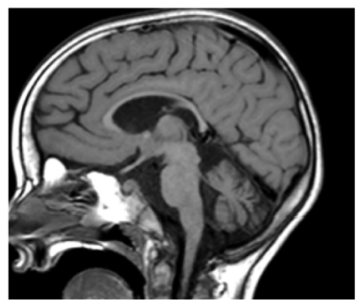

Patient 9 (6y)

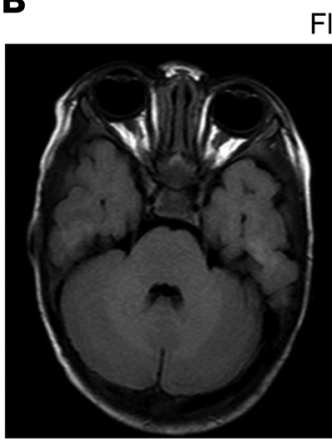

Flair


Patient $16(6 \mathrm{~m})$

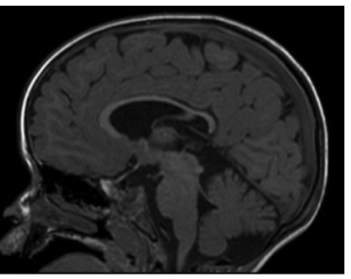

Patient $16(2.5 \mathrm{y})$
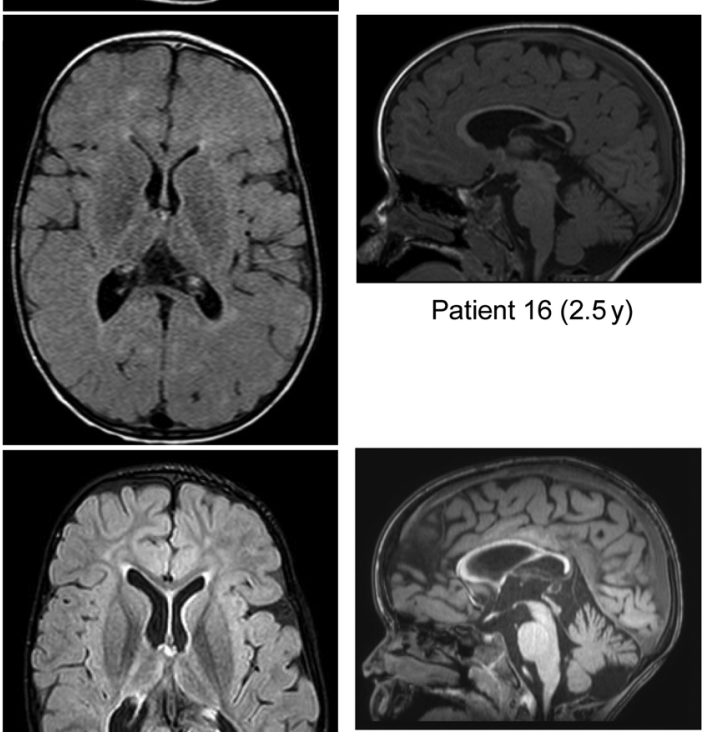

Patient 16 (4y)
Figure 3. Brain MRIs according to clinical severity and followup. (A) MRIs of patients with distinct clinical severity at the age of 6 years. Patient 7 (top row) shows the mildest presentation, with walking acquisition and spastic paraplegia. In FLAIR sequences, mild hyperintensities of the periventricular white matter (WM), with normal CC and internal capsule were observed. In T1 sagittal sequences, normal cerebellum and CC were observed. Patient 9 (bottom row) was able to hold the head but developed dystonia and spasticity with failure to thrive (-4 SD). Despite this severe clinical presentation, the FLAIR sequences show an abnormal hypersignal of the WM only in the periventricular and deep regions that are atrophic. The CC and the cerebellar vermis are atrophic. (B) Sequential MRIs of patient 16. At 6 months, all the WM structures including the cerebellum, the brainstem, and the CC appear unmyelinated. The $\mathrm{CC}$ is thin on the $\mathrm{T} 1$ sagittal section. A progression in the myelination has occurred at 2.5 years of age but the posterior part of the internal capsules is not myelinated, with periventricular hypersignals and an isosignal of the deep and subcortical WM and atrophic thalami. At 4 years of age the internal capsules and the deep and subcortical WM show normal myelinated signal, whereas the thalami and cerebellar vermis appeared as hypersignals and atrophic.

In an attempt to provide a therapeutic option for this life-threatening condition, we chose an FDAapproved drug targeting the de novo Cer biosynthesis pathway, fingolimod (FTY720). This pleiotropic drug is reported to act as an inhibitor of the enzyme prior to DEGS1, Cer synthase (CerS) (Figure 1) (29). We used concentrations of 3.3, 1.0, and $0.3 \mathrm{ng} / \mu \mathrm{l}$ FTY720, from birth to 120 hours postfertilization (hpf), without observing deleterious effects on survival. Also, the number of $\mathrm{MBP}^{+}$signals along the spinal cord was increased in MO-DEGS1 larvae after treatment, approaching the number of $\mathrm{MBP}^{+}$ cells in control larvae (Figure 5, F-M). The locomotor deficit of MO-DEGS1 larvae was remarkably ameliorated after 5 days of treatment with FTY720 at $1.0 \mathrm{ng} / \mu \mathrm{l}$ (Figure $6, \mathrm{~A}$ and $\mathrm{B}$ ). This recovery correlated with an amelioration of total DhCer levels and DhCer/Cer ratios upon treatment (Figure 6C). The effect of the drug was more marked towards some DhCer species (the saturated and monounsaturated C16-, C17-, C18-, C20-, C22, C24-, C26, and $\mathrm{C} 28$-DhCer ), which also accumulated to the greatest extent. Of note, a 10-fold decrease of C10Cer was also observed, and recovered with treatment (Supplemental Table 2). Next, we tested a potential protective effect of FTY720 on ROS generation in DEGS1 fibroblasts (patients 4, 7, and 9 as above), by using a dose of $5 \mu \mathrm{M}$ for 6 hours and measuring the ROS levels, as described above. FTY720 treatment prevented the elevation of ROS levels in patient fibroblasts (Figure 6D), while no effect was seen in control fibroblasts. These results provide proof of principle that FTY720 reduces the accumulation of DhCer and, thus, results in diminished ROS production at the cellular level. 


\section{A}

$\begin{array}{ll}\text { - Control fibroblasts } & \text { P7 (p.Asn255Ser) } \\ \left.\text { - P4 (p.Leu114Profs }{ }^{\star} 11 / p . A s n 255 S e r\right) & \text { A P9 (p.Asn113Asp) }\end{array}$
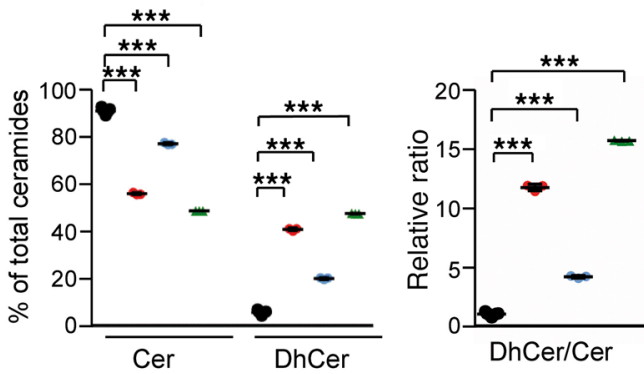

B
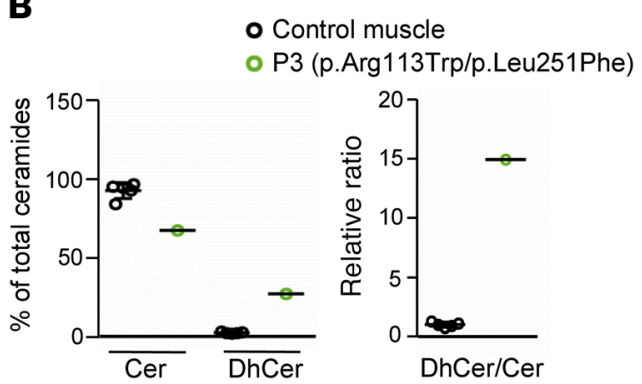

C

D
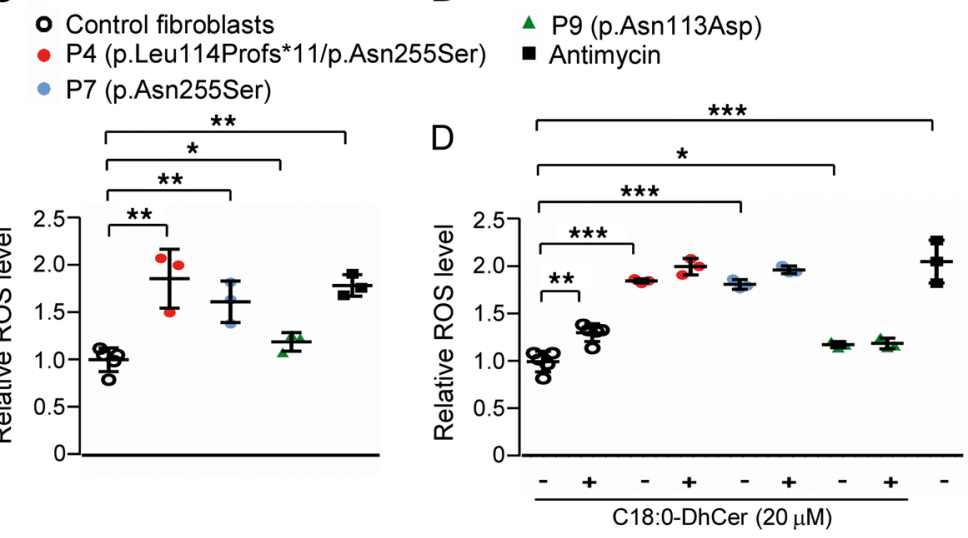

Figure 4. Dihydroceramide and ceramide imbalance in human patient fibroblasts and muscle. (A) Ceramides (Cer), dihydroceramides (DhCer), and the DhCer/Cer ratios in human controls $(n=9)$ and patient fibroblasts (P4, P7, and P9) $(n=3)$ and (B) in human controls $(n=5)$ and patient muscle $(\mathrm{P} 3)(n=1)$. Data are represented as percentage of total Cer and DhCer. (C) Intracellular ROS was quantified using the probe $\mathrm{H}_{2}$ DCFDA in patient fibroblasts (patients 4, 7, and 9) and controls $(n=5)$ at a basal level. (D) Exogenous DhCer (C18:0) treatment ( $20 \mu \mathrm{M}, 6$ hours) was applied to control and patient fibroblasts. Antimycin was used as positive control for ROS generation. The fibroblast results are from 3 independent experiments performed in triplicate. Data are shown as the mean $\pm \mathrm{SD}$. ${ }^{*} P<0.05 ;{ }^{*} P<0.01$; ${ }^{* *} P<0.001$ by 1-way ANOVA ( $\mathbf{A}$ and $\mathbf{C}$ ) or 2-way ANOVA followed by Tukey's post hoc test (D).

\section{Discussion}

This study highlights the interest of using freely accessible information-exchange platforms, such as GeneMatcher after exome sequencing, for rapid identification of molecular causes of ultrarare disorders and collection of sufficient cases to delineate a disease spectrum and improve management (30).

Patients with DEGS1-related LD shared clinical and MRI features of hypomyelinating LD. In this heterogeneous group of LDs, a large clinical spectrum of disease severity has been described in terms of defective myelination and axonal suffering. A large majority of our DEGS1 patients present the severe form of hypomyelinating LD, accompanied by thin CC and cerebellar atrophy as observed with TUBB4related LD (31). However, the hypomyelination observed in TUBB4, as well as in the severe forms of PLP1-related LD, is more diffuse and remains stable over time (32). The relative preservation of the subcortical white matter relative to the clinical severity of DEGS1 patients may represent an interesting MRI diagnostic feature. Likewise, the observed improvement in myelination in our patients contrasts with the progressive atrophy affecting first the CC, then thalami and cerebellum, and finally the whole brain. This suggests a critical role of DEGS1 and Cer metabolism in myelin development and maintenance, but also in neuronal/ axonal functioning. The hypomyelinating pattern of the deep white matter and the T2 hyperintensities of the thalamic regions are also observed in infantile GM1/ GM2 gangliosidosis $(22,33)$. Finally, the association of central and peripheral demyelination are the hallmarks of disorders of sphingolipid metabolism (MLD and GLD), and of the peroxisomes (34). Although individual features in the DEGS1-related patients are not specific, their combination results in a defined pattern distinguishable from other LDs.

The importance of maintaining sphingolipid balance for the nervous system is well exemplified in the lysosomal disorders such as Gaucher, Niemann Pick, or Farber, all one enzymatic step away from DEGS1 (Figure 1) (8). Indeed, in many lipid storage disorders, deficiency or malfunctioning of one of the enzymes involved in sphingolipid metabolism results in accumulation of the corresponding lipid substrate, leading to cellular dysfunction and death. Accumulation of DhCer is involved in many stress signals such as cell cycle regulation, autophagy induction, apoptosis, and ROS generation $(35,36)$, the latter being substantiated by our results. Redox imbalance is intertwined with energy homeostasis in diseases of myelin $(23,37)$, and may play a role in the cachexia observed in the large majority of our patients. Moreover, suppressing DEGS1 led to cell cycle arrest, cell growth inhibition, and apoptosis in cell culture models $(16,38)$, which may explain the decrease of myelinating $\mathrm{MBP}^{+}$oligodendrocyte numbers and perhaps contribute to the general developmental delay and impressive failure to thrive in our patients. Concerning the biogenesis and stability of the myelin sheath, an enhanced proportion of DhCer (in particular C16-DhCer) has recently been shown to destabilize model membranes by increasing permeability and the formation of rigid domains (35). Of note, C16-DhCer is increased in our MO-DEGS1 model and lowered by FTY720 treatment (Supplemental Table 2). It is therefore tempting to posit that the exquisitely regulated process of myelin sheath biogenesis and compaction, including the lipid-lipid and lipid-protein interactions with major protein players such as MBP, MAG, or PLP $(14,39)$, is likely to be severely 
A



MO-Control MO-DEGS1

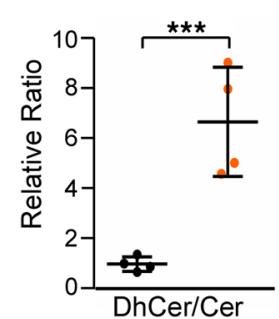

B



C



D

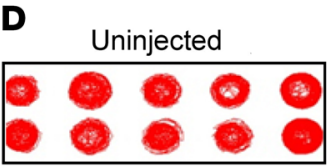

MO-Control

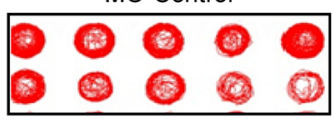

MO-DEGS1

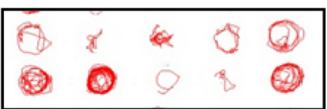

E

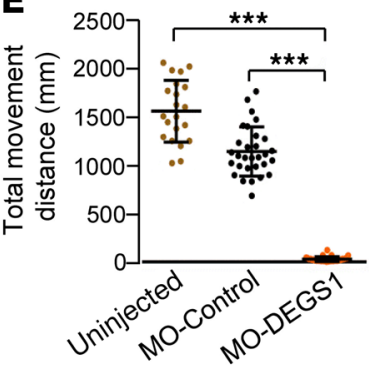

MO-Control
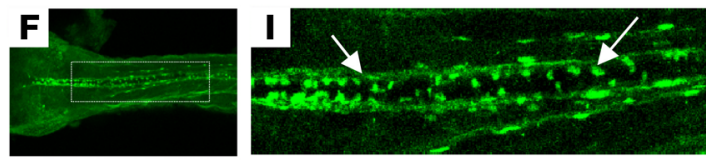

MO-DEGS1

MO-DEGS1+
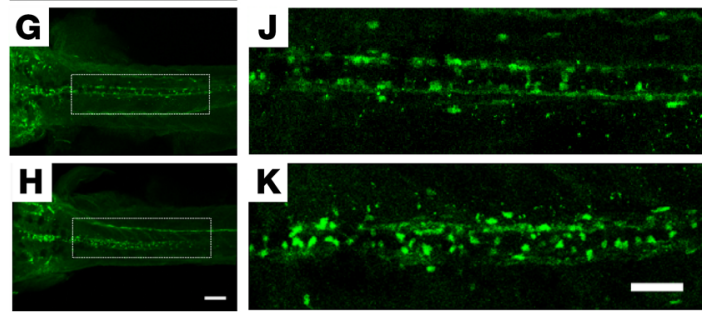

L

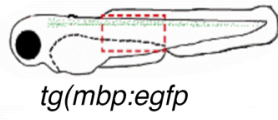

M

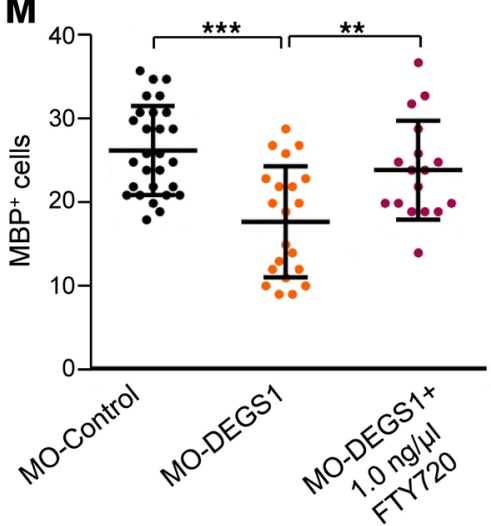

Figure 5. Impact of degs1 knockdown in Danio rerio larvae. (A) Ceramides (Cer), dihydroceramides (DhCer), and the DhCer/Cer ratios in MO-control and MO-DEGS1 zebrafish, $n=4 /$ condition (5 larvae per tube/condition), at $5 \mathrm{dpf}$. Data are represented as percentage of total Cer and DhCer. (B) Representative images of the normal, mild, and severe phenotypes observed in the MO-control- and MO-DECS1-injected groups. Scale bar: $100 \mu \mathrm{m}$. (C) Quantification at $5 \mathrm{dpf}$ of the percentage of normal (blue), mild (purple), and severe (orange) phenotypes groups obtained. Values are the percentage \pm SD of 3 independent experiments, with $n=50$ animals per group per experiment. (D) Examples of digital tracks of 10 single larvae of each condition shown in red. (E) Scatter plot displaying the total movement distance by different larvae: uninjected larvae $(n=20), 1,564.4 \pm 321.6 \mathrm{~mm}$; MO-control $(n=30), 1,146.6 \pm 255.2 \mathrm{~mm}$; and MO-DEGS1 ( $n=33), 28.3 \pm 24.5 \mathrm{~mm}$. (F-H) Dorsal views of larvae injected with MO-control and MO-DEGS1, and (I-K) inserts of boxed areas at $4.5 \mathrm{dpf}$. Scale bar: $100 \mu \mathrm{m}$. White arrows indicate $\mathrm{MBP}^{+}$cells (myelinating oligodendrocytes). (L) Illustration of a tg(mbp:egfp) larva. (M) Scatter plot displaying the number of $\mathrm{MBP}^{+}$cells in the dorsal spinal cord of 4.5-dpf larvae (MO-control, $n=30$; MO-DEGS1, $n=28$; MO-DEGS1 $+1 \mathrm{ng} / \mu \mathrm{l} \mathrm{FTY720,} n=19$ ). Data are shown as the mean $\pm \mathrm{SD}$. ${ }^{*} P<0.05 ;{ }^{* *} P<0.01 ;{ }^{* *} P<0.001$ by 2 -tailed unpaired Student's $t$ test (A) or 1-way ANOVA followed by Tukey's post hoc test (E and $\left.\mathbf{M}\right)$.

disturbed, simply by the biophysical consequences of the DhCer/ Cer imbalance on the cellular membranes.

Further, it has been recently shown that in the Drosophila (ifc) $\mathrm{KO}$, photoreceptor degeneration was caused by increased DhCer, not reduced levels of Cer (20). Along the same lines, Cer reduction by itself appears to have a positive effect by enhancing myelin repair in acute and chronic demyelination paradigms, as deduced from a model of acid sphingomyelinase deficiency (40). Of note, impairment of alkaline ceramidase 3 (ACER3), just one step after
DEGS1 (Figure 1), causes a recently described form of childhood leukoencephalopathy of similar clinical presentation to the DEGS1-related LD, with a hypomyelinating aspect of the periventricular and deep white matter, severe brain atrophy by 5 years of age, and peripheral neuropathy (5). It is intriguing that the plasma profile of the described patient showed mainly accumulation of DhCer, along with various Cer forms and lactosylceramides to a lesser extent (5). Further investigations will be necessary to determine the precise molecular mechanisms through which excess of 
A



C

- MO-Control

o MO-Control + 1 ng/ $\mu$ I FTY720



MO-DEGS1

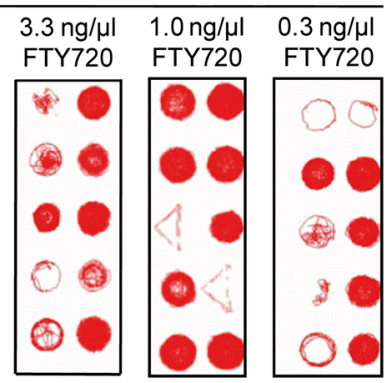

B

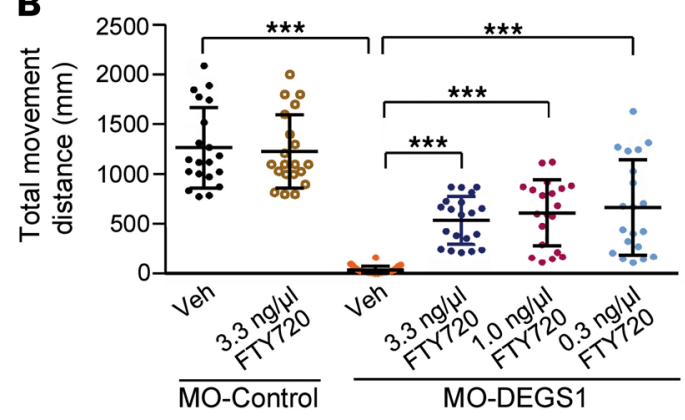

MO-DEGS1

- MO-DEGS1 + $1 \mathrm{ng} / \mu \mathrm{l}$ FTY720



D

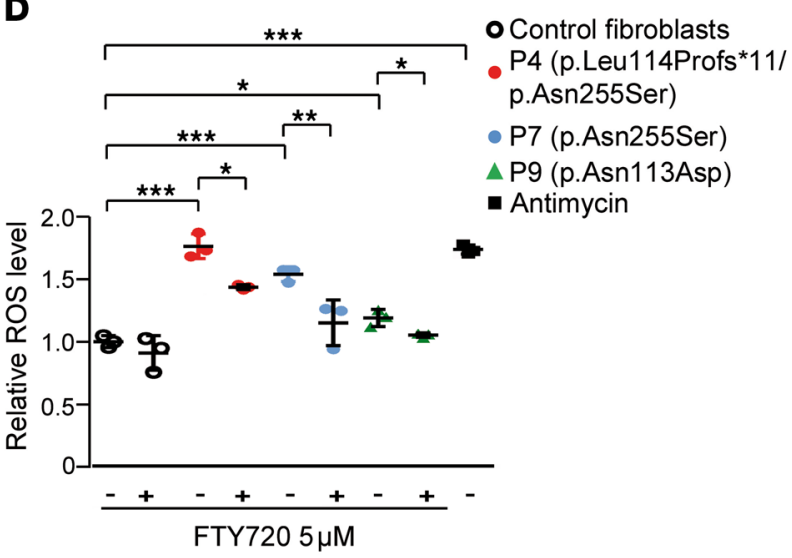

Figure 6. FTY720 ameliorates the locomotor, biochemical, and cellular phenotypes in MO-DEGS1 larvae and lowers ROS levels in patient fibroblasts. (A) MO-DEGS1 larvae were treated for 120 hours with vehicle (Veh) or fingolimod (FTY720) at 0.3, 1.0, and 3.3 ng/ $\mu$ l. Digital tracks of larvae are shown in red. (B) Scatter plot ( $n=20$ per condition) showing total movement distance $(\mathrm{mm})$ upon FTY720 treatment. Total movement distance (mm) traveled by MO-control larvae (vehicle, 1,268.2 $\pm 402.9 \mathrm{~mm}$; FTY720, 1,230 $\pm 365.2 \mathrm{~mm}$ ), compared with MO-DEGS1 (vehicle, $44.6 \pm 39.2 \mathrm{~mm}$ ) and the different treat-

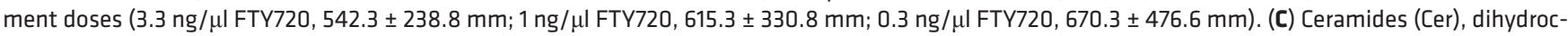
eramides (DhCer), and the DhCer/Cer ratios in MO-control and MO-DEGS1 zebrafish treated with $1 \mathrm{ng} / \mu \mathrm{l} \mathrm{FTY720} \mathrm{(} n=4$ [5 larvae per tube/condition] larvae/ condition at $5 \mathrm{dpf}$ ). Data are represented as percentage of total Cer and DhCer. The results are from 3 independent experiments. (D) Intracellular ROS is partially normalized by FTY720 in patient fibroblasts $(n=3)$ compared with controls $(n=3)$. Data are shown as the mean \pm SD. ${ }^{*} P<0.05$; ${ }^{* *} P<0.01$; ${ }^{* * *} P<0.001$ by 2 -way ANOVA followed by Tukey's post hoc test.

DhCer may be toxic for myelinating oligodendrocytes or for the biogenesis of the myelin sheath. By the same token, and beyond the plausible direct negative effects of substrate accumulation in this case, we should acknowledge that sphingolipid metabolism constitutes an intricate network coupled in a metabolic ripple (16). As such, the loss of a given enzyme function may cause a shift in the substrate specificity of other enzymes in the pathway, leading to increased production of atypical lipid products that may be toxic to the cell (41). This intriguing possibility should be explored for DEGS1, likely by using a systems biology type of approach, ideally including integration of comprehensive lipidomics and transcriptomics data (42). A bulk of evidence suggests that FTY720 has potential to become a lead player in this context. Its success in relapsing-remitting MS relies on its activity as immunosuppressant, acting by sequestering circulating mature lymphocytes to the lymph nodes, and preventing their penetration into the brain and subsequent myelin destruction. Its molecular effects promoting neuron and oligodendrocyte survival are based on the structural similarity of FTY720 to sphingosine, which allows it to work as a bona fide functional antagonist to S1P receptors $(43,44)$, and has lately been shown to even improve microstructural integrity and myelination in the white matter tracts of human MS patients (45).
In part because of this structural similarity to sphingosine, FTY720 is a pleiotropic drug that also interferes with sphingolipid de novo biosynthesis, mainly by inhibiting CerS $(29,43)$, the enzyme that converts sphinganine to DhCer, one step prior to DEGS1, and also converts sphingosine to Cer (Figure 1). There are 66 CerS orthologs in mammals (46) and 9 - due to whole genome duplication - in zebrafish (47). These enzymes transfer a variable length fatty acyl-coenzyme A (fatty acyl-CoA) to the amine group of sphinganine or sphingosine. Studies employing genetic manipulations have demonstrated that different CerS isoforms exhibit strong preference for fatty acyl-CoAs with differing carbon chain lengths, although with extensive overlaps. CerS1 exclusively uses 18-carbon (C18) fatty acids, forming C18 (d18:1/18:0) Cer, while CerS2 preferentially forms d18:1/24:0 (C24:0) and d18:1/24:1 (C24:1) Cer. CerS3 synthesizes very longchain Cer (>C26-Cer), CerS4 forms C18-/C20-/C24-Cer, and CerS5 and CerS6 synthesize mostly C14-/C16-/C18-Cer (48, 49). Thus, the broad spectrum of DhCer species accumulated first in the DEGS1 zebrafish model, and then reduced by FTY720 (spanning C13:1 DhCer to C28:0 DhCer, Supplemental Table 2), adds complexity to future studies aiming to identify the drug's most plausible targets in this model. 
Additional experimental evidence supports the protective effect of FTY720 in sphingolipid metabolism-related disorders, such as GLD, which is caused by inactivation of galactosylceramidase (GALC) resulting in the accumulation of the toxic metabolite galactosylsphingosine (psychosine) in the brain. FTY720 treatment attenuated the psychosine-induced demyelination process in mouse organotypic cerebellar slice cultures (50). With this notion in mind, and our proof-of-principle results in a model organism, we posit that FTY720 (or novel, more specific next-generation sphingosine analogs interfering with CerS or other enzymatic steps along the pathway) may provide a promising therapeutic option for white-matter disorders linked to DEGS1 deficiency and perhaps others in sphingolipid metabolism, paving the way for the repurposing of FTY720 for other diseases. Our next step as a DEGS1 consortium is the validation of a robust biomarker in plasma (such as DhCer/Cer ratios) to monitor target engagement upon treatment and to prepare for early clinical intervention aiming to alleviate and modify the disease course of this life-threatening condition.

\section{Methods}

\section{Patients, genetic studies, and ethics statement}

All 19 affected individuals underwent extensive clinical examination by a pediatric neurologist and at least one expert clinical geneticist at their referral hospitals, where broad metabolic and genetic testing was performed. Standardized phenotypic data were collected by review of the clinical histories and followup investigations (see Tables 1-5). All available clinical and MRI data were collected and jointly reviewed. WES on the Illumina HiSeq platform was performed according to the following paradigms: (a) trio-based clinical diagnostic WES (individuals 1, 7, 9, 10, 11, 14, and 17); (b) trio-based WES or trio-based WES plus affected sibling in a research-based analysis (individuals 3, 4, 15, 18 , and 19); or (c) WES of an affected individual followed by single-site testing in parental DNA samples (individuals 6 and 8). Point mutations and indels were confirmed by Sanger sequencing of DNA samples from all available family members, when possible (Supplemental Table 3). The exome sequences have been deposited in public repositories: patient 4 (phs000711.v5.p1), patient 13 (phs000744.v4.p2), and patient 18 (phs001272.v1.p1) into dbGaP (https://www.ncbi.nlm. nih.gov/gap); patient 1 (P0007232), patient 6 (P0005949), patient 7 (P0007704), patient 8 (P0007705), patient 9 (P0007706), patient 10 (P0007707), and patient 15 (P0007703) into Phenome Central (https://www.phenomecentral.org/).

\section{Targeted lipidomics profiling}

Briefly, cells were harvested using trypsin, and cell pellets contained 3.5 million cells. Muscle biopsy samples (50 mg) were snap frozen and stored at $-80^{\circ} \mathrm{C}$ until extraction. The zebrafish samples consisted of a pool of 5 zebrafish larvae ( $5 \mathrm{dpf})$ per experimental point $(n=4)$ per condition. Lipidomics experiments were performed at Biocrates Life Science AG, as follows. Fibroblast cells were lysed by freeze-thaw cycles and metabolites were extracted with $60 \mu \mathrm{l}$ ice-cold methanol. Zebrafish larvae were homogenized using Precellys and metabolites were extracted with $60 \mu \mathrm{l}$ ice-cold methanol. For measuring metabolite concentrations from muscle tissue, the samples were first weighed and then homogenized using Precellys in methanol. Samples were centrifuged and the supernatant was used for further analysis. Cer, DhCer, and 2-hydroxyacyl Cer were quantitatively analyzed by high-throughput flow injection analysis electrospray ionization tandem mass spectrometry (FIA-ESI-MS/MS). The reported lipid annotation represents a sum signal of all isobaric lipids with the same molecular weight ( \pm 0.5 Da range) within the same lipid class. Multiple reaction monitoring (MRM) detection in positive and negative mode was performed using a 4000 QTRAP (SCIEX) instrument. Preparation of 20- $\mu$ l samples was followed by a methanol/ $\mathrm{CHCl}_{3}$ liquid/liquid extraction protocol. In addition to 5 internal standards to compensate for matrix effects, 43 external standards were used for a multipoint calibration. The quantitative data analysis was performed with our in-house software MetIDQ, enabling isotopic correction. All preanalytical and analytical procedures were performed, documented, and reviewed according to our ISO 9001:2008-certified in-house quality management rules and guidelines.

\section{Zebrafish strains}

Zebrafish embryos were obtained by mating of adult fish using standard methods. All fish strains were maintained individually as inbred lines. The wild-type zebrafish strain was AB/Tu (RRID: ZIRC_ZL1/ RRID: ZIRC_ZL57), and Tg(mbp:egfp) transgenic zebrafish were generated as described previously (27), in order to visualize myelinating oligodendrocytes in living zebrafish (Zfin, https://zfin.org). Embryos were reared at $28.5^{\circ} \mathrm{C}$ until processing for analyses at desired stages.

\section{ISH and immunolabeling}

Whole-mount ISH was performed with standard protocols, as described previously (51). Embryos were fixed in $4 \%$ paraformaldehyde (PFA), cryoprotected in 30\% sucrose, and embedded in OCT. Blocks were frozen to improve tissue preservation, and then $10-\mu \mathrm{m}$ sections were cut on a Leica CM1510-1 cryostat. Hybridization was performed at $65^{\circ} \mathrm{C}$ with digoxigenin-labeled RNA probes. The antisense digoxigenin-labeled RNA probe for zebrafish degs1 (ENSDART00000013007.5) was generated by in vitro transcription of an amplicon from exon 1 (primers, forward 5'-ATGGGGAACCGCGTGGCGCG-3'; reverse, 5'-TCACTCCTGCTTGACGTCTC-3'). Following purification, the fragment was subcloned into a pBluescript dual promoter vector and was sequenced to confirm identity. The construct was linearized with BamHI, and digoxigenin-labeled antisense mRNA probe was generated by $\mathrm{T} 3$ in vitro transcription using the DIG-RNA Labeling Kit (Roche, 11-175-025-910). No signal was detected using the sense probe.

For EGFP immunolabeling, staged embryos were fixed in $4 \%$ PFA at room temperature for 20 minutes, washed in 0.1\% Tween 20/ PBS, and incubated overnight at $4^{\circ} \mathrm{C}$ with anti-GFP primary antibody (GFP102; 1:500; Aves Labs) in blocking solution followed by secondary antibodies conjugated with Alexa Fluor 488. Sections were then washed 3 times in $1 \times$ PBS and mounted in Fluoromount (MilliporeSigma). Cryostat sections were imaged on a Nikon ECLIPSE 80i fluorescence microscope. ISH and fluorescence images were processed using Image (NIH). Confocal images were acquired using a TCS SL laser scanning confocal spectral microscope (Leica Microsystems). Calculation of the number of $\mathrm{MBP}^{+}$cells was performed using plugins in Image J software. We imaged a fixed area in the spinal cord (dotted box in Figure $5, \mathrm{~F}-\mathrm{H}$ ). $\mathrm{MBP}^{+}$cells (myelinating oligodendrocytes) were counted in the dorsal spinal cord of 4.5-dpf larvae. 


\section{Antisense $\mathrm{MO}$ injections}

For MO knockdowns, embryos were injected with splice-blocking MOs obtained from GeneTools LLC. MO-degs1, 5'-GCTGAATAACTGCTCTCACCATTGG-3' (herein referred to as MO-DEGS1), was designed as complementary to the genomic sequence flanking the exon 2/intron 2 boundary. Briefly, $1 \mathrm{nl}$ of $5 \mathrm{ng} / \mathrm{nl}$ or $10 \mathrm{ng} / \mathrm{nl}$ MO-control and MO-DEGS1 was injected into the yolk of 1-cell-stage embryos. After injection, embryos were incubated at $28.5^{\circ} \mathrm{C}$ until the desired stage was reached. Injected embryos and uninjected clutchmate controls were analyzed from 0 to $5 \mathrm{dpf}$. Every day, dead larvae were removed and the fraction of surviving larvae was recorded until 5 dpf. In order to verify the efficiency of the MOs, RNA from uninjected, MO-control-, and MO-DEGS1-injected larvae was extracted with TRIzol (Thermo Fisher Scientific), and reverse transcription was performed by first-strand cDNA synthesis with the QuantiTect Reverse Transcription kit (Qiagen). The targeted region of degs 1 was PCR amplified using primers complementary to sites in flanking exons located in exon 2 (5'-GGCTCTCTGAACCTGCTGAC-3') and exon 3 (5'-CTTGACGTCTCCGACCAGTT-3') using standard PCR conditions (annealing temperature: $60^{\circ} \mathrm{C}$ ), and migrated by electrophoresis in a $1 \%$ agarose gel; bands were excised, gel purified using a QIAquick gel extraction kit (Qiagen), and resulting clones were Sanger sequenced to confirm aberrant splicing events induced by the MO (Supplemental Figure 4, G-I; see complete unedited blots in the supplemental material.). The standard MO-control against $\beta$-globin was also obtained from GeneTools (5'-CCTCTTACCTCAGTTACAATTTATA-3') and was used as negative control.

\section{Larval locomotor behavior assay}

By 5 dpf, zebrafish larvae perform spontaneous swimming and their visual system is fully developed. Uninjected, MO-control, and MO-DEGS1 120-hpf larvae were transferred to individual wells of a 96-well plate with $150 \mu \mathrm{l}$ fresh fish water and allowed to acclimate for a few hours. The zebrafish larvae behavior was tracked and analyzed by EthoVision XT software and a DanioVision device from Noldus Information Technologies. This closed system consists of a camera placed above a chamber with circulating water and a temperature sensor that is set at $28.5^{\circ} \mathrm{C}$. Individualized larvae in a 96-well plate were placed in the chamber, which can provide different stimuli (light/dark environment, tapping, sound) controlled by the software. Prior to each experiment, larvae were left for 10 minutes in the dark for acclimation, followed by presentation of a predetermined series of alternating dark and light environment to the larvae. The natural locomotor behavior of zebrafish larvae is characterized by high activity in darkness and immobility in light environments. The final 25-minute-long experimental protocol was divided into a 5-minute period of darkness, and then a twicerepeated cycle of 5 minutes of bright light followed by 5 minutes of darkness. During the behavioral trial the total distance moved by every larva was measured. We obtained the distance moved and the mobility parameters from EthoVision tracking program analysis, and baseline parameters were subtracted out prior to the assay. All locomotor assays were performed at $1 \mathrm{pm}$ onwards to ensure steady activity of zebrafish (52). After observations, all subjects were euthanized with a lethal concentration of MS-222 tricaine (ethyl-3-aminobenzoate methanesulfonate salt; MilliporeSigma). Swimming behavior after drug treatment was performed by adding FTY720 to standard fish water to a final concentration of $3.3,1.0$, and $0.3 \mathrm{ng} / \mu \mathrm{l}$. DMSO was used as vehicle.
MO-control and MO-DEGS1 embryos (O hpf) were treated with FTY720 until $120 \mathrm{hpf}$ and the behavior assay was performed as described above. All measures were averaged across larvae within each condition $(n=20$ larvae/condition) and are reported as population means \pm SD.

\section{Statistics}

All experiments were repeated at least 3 times with consistent results. All cell culture data were from biologic triplicates, unless otherwise indicated. Statistical analysis was performed with 2-tailed, unpaired Student's $t$ test for comparison of 2 groups, or ANOVA followed by Tukey's post hoc test for multiple comparisons after verifying normality. Statistical analyses were performed using the GraphPad Prism 7.0 program. $P$ less than 0.05 was considered significant $\left({ }^{*} P<0.05,{ }^{* *} P<\right.$ $\left.0.01,{ }^{* * *} P<0.001\right)$.

\section{Study approval}

Human study. Human genetic studies conducted in research laboratories were approved by local ethics committees from participating centers: IDIBELL (PR076/14); INSERM U1163 (2015-03-03/DC 2014-2272); LEUKOFRANCE (CPP AU788, CNIL 1406552, AFSSAPS B90298-60); University of California, San Diego (IRB 140028), Peking University First Hospital ([2005]004), Mayo Clinic (IRB 12-009346), Johns Hopkins School of Medicine (NA_00045758); McMaster Children's Hospital (14-595-T), and Shahid Chamran University of Ahvaz (EE/97.24.317654/scu.ac.ir). Parental (or legal guardian) written informed consent was obtained for all affected children. Written informed consent was provided for the video appearing in the supplemental material.

Zebrafish study. All protocols used have been approved by the Institutional Animal Care and Use Ethic Committee (Barcelona Biomedical Research Park [PRBB]-IACUEC) and implemented according to national and European regulations. All experiments were carried out in accordance with the principles of the 3Rs.

\section{Author contributions}

DCP, NL, SF, JJ, MR, C. Cornet, D. Rubbini, JT, and CP performed and/or interpreted or supervised aspects of the different experiments. SAA, MEY, C. Casasnovas, MJW, DV, GL, KSP, I. Desguerre, HY, JW, MB, LB, MT, KNJ, DM, MSZ, MCP, BCL, EWK, FPV, EW, NLMS, JSC, RM, HG, NM, GS, LC, D. Rodriguez, JGG, $\mathrm{AF}$, and OBT undertook clinical and genealogical studies and provided clinical data and biological specimens from patients. I. Dorboz and AS undertook variant calling and bioinformatic analyses of WES data. DCP and AP analyzed and interpreted data and wrote the initial draft of the manuscript. OBT supervised the clinical and MRI data in coordination with AP. AP provided overall study direction, funding, coordination, supervision, and revised the manuscript. All authors critically reviewed the manuscript and approved the final version.

\section{Acknowledgments}

We thank the CERCA Program/Generalitat de Catalunya for institutional support. We also thank Juanjo Martínez and Cristina Guilera for excellent technical assistance and Asociación Española contra las Leucodistrofias (ALE-ELA España). We are indebted to the NIH NeuroBioBank for supplying the case material used for the human studies. The authors thank the affected individuals and 
their families for participation in this study. This study was supported by the ISCIII (FIS PI14/00581) (cofunded by the European Regional Development Fund); 'La Marató de TV3' Foundation 345/C/2014 (to AP, C. Casasnovas, and CP); the Hesperia Foundation and CIBER on Rare Diseases (CIBERER) (ACCI14-759) and the Secretariat for Universities and Research of the Ministry of Business and Knowledge of the Government of Catalonia (2017SGR1206) to AP; the Spanish Ministry of Economy and Competitiveness (MINECO-FEDER) (BFU2015-67400-P) to CP; the Spanish Institute for Health Carlos III (Miguel Servet program CPII16/00016) to SF; CIBERER to NL and MR; and a predoctoral fellowship awarded to DP from the Government of Catalonia through L'Agència de Gestió d'Ajuts Universitaris i de Recerca (AGAUR; FI-DGR 2014). XJ acknowledges a Sara Borrell postdoctoral fellowship from Instituto de Salud Carlos III. The National Research Agency (ANR-10-IAHU-01) and Fondation pour la Recherche Médicale (DEQ20160334938) provided grants to LC. The authors thank the members of LEUKOFRANCE, particularly the Biobank in Clermont Ferrand (Eleonore Pierre and Philippe Vago) and Kremlin Bicetre (Elise Lebigot and Abdelhamid Slama) University Hospitals. The European Union FP7 RD-Connect project, ELA Foundation (2009-007I4AV2), and "Les amis de Ianis" association (Ploudaniel, France) provided grants to OBT. I. Dorboz was supported by an ELA grant and is currently supported by the RD-connect project. JGG is supported by NIH grants P01HD070494, 1R01NS098004, R01NS048453, R01NS052455, and UL1TR001866 from the National Center for Advancing Translational Sciences (NCATS), the NIH Clinical and Translational Science Award (CTSA) program, the Simons Foundation Autism Research Initiative (grant 275275), Howard Hughes Medical Institute, and the Qatar National Research Foundation (NPRP 6-1463-3-351). We thank the Yale University Center for Mendelian Genomics (Mark Gerstein, Murat Gunel, Richard P. Lifton, Shrikant M. Mane, 5UM1HG006504) and the Broad Institute (Daniel G. MacArthur and Heidi L. Rehm,
5UM1HG008900) for sequencing support. NS was funded by NIH grant 1U54 HG006542. The Baylor-Hopkins Center for Mendelian Genomics and GeneMatcher are supported by a grant from the National Human Genome Research Institute (1U54HG006493). AF was supported by a Kennedy Krieger Institute Intellectual and Developmental Disabilities Research Center grant (funded through NICHD U54 HD079123). MB was funded by NIH grant NSO56780 and the National Ataxia Foundation. MCP was funded by grants from the NIH (NS 65768-01), the Peggy Furth Fund, and the National MS Society. EWK was funded by the Mayo Clinic Center for Individualized Medicine (CIM), the Investigative and Functional Genomics Program, and the William O. Lund, Jr. and Natalie C. Lund Charitable Foundation. Genetic testing was performed under the Care4Rare Canada Consortium funded by Genome Canada, the Canadian Institutes of Health Research, the Ontario Genomics Institute, Ontario Research Fund, Genome Quebec, and Children's Hospital of Eastern Ontario Foundation (to MT). We wish to acknowledge the contribution of the high-throughput sequencing platform of the McGill University and Génome Québec Innovation Centre, Montréal, Canada. We are grateful to Cheol-Hee Kim for providing the Tol2 transposon vector, and Bellvitge Biobank for providing control human muscle tissues. AP and EWK are members of the Undiagnosed Diseases Program International (UDNI). The views expressed herein are the authors' and do not necessarily reflect the official policy or position of the Department of the Navy, Department of Defense, or the US Government.

Address correspondence to: Aurora Pujol, Neurometabolic Diseases Laboratory, IDIBELL, Hospital Duran i Reynals, Gran Via 199, 08908 L'Hospitalet de Llobregat, Barcelona, Spain. Phone: 34.932607137; Email: apujol@idibell.cat. Or to: Odile Boespflug-Tanguy, INSERM UMR 1141, DHU PROTECT, Paris Diderot University, Sorbonne Paris Cité, France; Phone: 33.140034170; Email: odile.boespflug-tanguy@aphp.fr.
1. Köhler W, Curiel J, Vanderver A. Adulthood leukodystrophies. Nat Rev Neurol. 2018;14(2):94-105.

2. Parikh S, et al. A clinical approach to the diagnosis of patients with leukodystrophies and genetic leukoencephelopathies. Mol Genet Metab. 2015;114(4):501-515.

3. van der Knaap MS, Bugiani M. Leukodystrophies: a proposed classification system based on pathological changes and pathogenetic mechanisms. Acta Neuropathol.2017;134(3):351-382.

4. Dorboz I, et al. Biallelic mutations in the homeodomain of NKX6-2 underlie a severe hypomyelinating leukodystrophy. Brain. 2017;140(10):2550-2556.

5. Edvardson S, et al. Deficiency of the alkaline ceramidase ACER3 manifests in early childhood by progressive leukodystrophy. J Med Genet. 2016;53(6):389-396.

6. Mendes MI, et al. Bi-allelic mutations in EPRS, encoding the glutamyl-prolyl-aminoacyl-tRNA synthetase, cause a hypomyelinating leukodystrophy. Am J Hum Genet. 2018;102(4):676-684.

7. Eichler F, et al. Hematopoietic stem-cell gene therapy for cerebral adrenoleukodystrophy. N Engl JMed. 2017;377(17):1630-1638.
8. Ferreira CR, Gahl WA. Lysosomal storage diseases. Transl Sci Rare Dis. 2017;2(1-2):1-71.

9. Endo K, Akiyama T, Kobayashi S, Okada M. Degenerative spermatocyte, a novel gene encoding a transmembrane protein required for the initiation of meiosis in Drosophila spermatogenesis. Mol Gen Genet. 1996;253(1-2):157-165.

10. Ternes P, Franke S, Zähringer U, Sperling P, Heinz E. Identification and characterization of a sphingolipid delta 4-desaturase family. J Biol Chem. 2002;277(28):25512-25518.

11. Hannun YA, Obeid LM. Many ceramides. J Biol Chem. 2011;286(32):27855-27862.

12. Montefusco DJ, et al. Distinct signaling roles of ceramide species in yeast revealed through systematic perturbation and systems biology analyses. Sci Signal. 2013;6(299):rs14.

13. Rodriguez-Cuenca S, Barbarroja N, Vidal-Puig A. Dihydroceramide desaturase 1, the gatekeeper of ceramide induced lipotoxicity. Biochim Biophys Acta. 2015;1851(1):40-50.

14. Schmitt S, Castelvetri LC, Simons M. Metabolism and functions of lipids in myelin. Biochim Biophys Acta. 2015;1851(8):999-1005.

15. Yeh YY. Long chain fatty acid deficits in brain myelin sphingolipids of undernourished rat pups. Lipids. 1988;23(12):1114-1118.

16. Hannun YA, Obeid LM. Sphingolipids and their metabolism in physiology and disease. Nat Rev Mol Cell Biol. 2018;19(3):175-191.

17. Di Pardo A, et al. De novo synthesis of sphingolipids is defective in experimental models of Huntington's disease. Front Neurosci. 2017;11:698.

18. Fonteh AN, Ormseth C, Chiang J, Cipolla M, Arakaki X, Harrington MG. Sphingolipid metabolism correlates with cerebrospinal fluid Beta amyloid levels in Alzheimer's disease. PLOS ONE. 2015;10(5):e0125597.

19. Holland WL, et al. Inhibition of ceramide synthesis ameliorates glucocorticoid-, saturated-fat-, and obesity-induced insulin resistance. Cell Metab. 2007;5(3):167-179.

20. Jung WH, et al. Lipophagy prevents activitydependent neurodegeneration due to dihydroceramide accumulation in vivo. EMBO Rep. 2017;18(7):1150-1165.

21. Sobreira N, Schiettecatte F, Valle D, Hamosh A. GeneMatcher: a matching tool for connecting investigators with an interest in the same gene. Hum Mutat. 2015;36(10):928-930. 
22. Steenweg ME, et al. Magnetic resonance imaging pattern recognition in hypomyelinating disorders. Brain. 2010;133(10):2971-2982.

23. López-Erauskin J, et al. Impaired mitochondrial oxidative phosphorylation in the peroxisomal disease X-linked adrenoleukodystrophy. Hum Mol Genet. 2013;22(16):3296-3305.

24. Enomoto A, Omae F, Miyazaki M, Kozutsumi Y, Yubisui T, Suzuki A. Dihydroceramide:sphinganine C-4-hydroxylation requires Des2 hydroxylase and the membrane form of cytochrome b5. Biochem J. 2006;397(2):289-295.

25. Omae F, Miyazaki M, Enomoto A, Suzuki A. Identification of an essential sequence for dihydroceramide C-4 hydroxylase activity of mouse DES2. FEBS Lett. 2004;576(1-2):63-67.

26. Czopka T. Insights into mechanisms of central nervous system myelination using zebrafish. Glia. 2016;64(3):333-349.

27. Jung SH, et al. Visualization of myelination in GFP-transgenic zebrafish. Dev Dyn. 2010;239(2):592-597.

28. Baraban SC, Dinday MT, Hortopan GA. Drug screening in Scn1a zebrafish mutant identifies clemizole as a potential Dravet syndrome treatment. Nat Commun. 2013;4:2410.

29. Lahiri S, Park H, Laviad EL, Lu X, Bittman R, Futerman AH. Ceramide synthesis is modulated by the sphingosine analog FTY720 via a mixture of uncompetitive and noncompetitive inhibition in an Acyl-CoA chain length-dependent manner. J Biol Chem. 2009;284(24):16090-16098.

30. [No authors listed]. Rare advances for rare diseases. Lancet Neurol. 2017;16(1):1.

31. Tonduti D, et al. TUBB4A-related hypomyelinating leukodystrophy: New insights from a series of 12 patients. Eur J Paediatr Neurol. 2016;20(2):323-330.

32. Sarret C, et al. Time-course of myelination and atrophy on cerebral imaging in 35 patients with PLP1-related disorders. Dev Med Child Neurol. 2016;58(7):706-713.

33. Sandhoff K, Christomanou H. Biochemistry and genetics of gangliosidoses. Hum Genet. 1979;50(2):107-143.

34. Kohlschütter A. Lysosomal leukodystrophies: Krabbe disease and metachromatic leukodystrophy. Handb Clin Neurol. 2013;113:1611-1618.

35. Hernández-Tiedra S, et al. Dihydroceramide accumulation mediates cytotoxic autophagy of cancer cells via autolysosome destabilization. Autophagy. 2016;12(11):2213-2229.

36. Siddique MM, Li Y, Chaurasia B, Kaddai VA, Summers SA. Dihydroceramides: From bit players to lead actors. J Biol Chem. 2015;290(25):15371-15379.

37. Ruiz M, et al. Oxidative stress and mitochondrial dynamics malfunction are linked in Pelizaeus-Merzbacher disease. Brain Pathol. 2018;28(5):611-630.

38. Kraveka JM, et al. Involvement of dihydroceramide desaturase in cell cycle progression in human neuroblastoma cells. J Biol Chem. 2007;282(23):16718-16728.

39. Olsen ASB, Færgeman NJ. Sphingolipids: membrane microdomains in brain development, function and neurological diseases. Open Biol. 2017;7(5):170069.

40. Chami M, et al. Acid sphingomyelinase deficiency enhances myelin repair after acute and chronic demyelination. PLOS ONE. 2017;12(6):e0178622.

41. Penno A, et al. Hereditary sensory neuropathy type 1 is caused by the accumulation of two neurotoxic sphingolipids. J Biol Chem. 2010;285(15):11178-11187.

42. Ruiz M, et al. Altered glycolipid and glycerophospholipid signaling drive inflammatory cascades in adrenomyeloneuropathy. Hum Mol Genet.
2015;24(24):6861-6876.

43. Brunkhorst R, Vutukuri R, Pfeilschifter W. Fingolimod for the treatment of neurological diseases-state of play and future perspectives. Front Cell Neurosci. 2014;8:283.

44. Miron VE, Jung CG, Kim HJ, Kennedy TE, Soliven B, Antel JP. FTY720 modulates human oligodendrocyte progenitor process extension and survival. Ann Neurol. 2008;63(1):61-71.

45. Gurevich M, Waknin R, Stone E, Achiron A. Fingolimod-improved axonal and myelin integrity of white matter tracts associated with multiple sclerosis-related functional impairments. CNS Neurosci Ther. 2018;24(5):412-419.

46. Levy M, Futerman AH. Mammalian ceramide synthases. IUBMB Life. 2010;62(5):347-356.

47. Brondolin $\mathrm{M}$, et al. Identification and expression analysis of the zebrafish homologs of the ceramide synthase gene family. Dev Dyn. 2013;242(2):189-200.

48. Ben-David O, Futerman AH. The role of the ceramide acyl chain length in neurodegeneration: involvement of ceramide synthases. Neuromolecular Med. 2010;12(4):341-350.

49. Mizutani Y, Kihara A, Igarashi Y. Mammalian Lass 6 and its related family members regulate synthesis of specific ceramides. Biochem J. 2005;390(Pt 1):263-271.

50. O'Sullivan C, Dev KK. Galactosylsphingosine (psychosine)-induced demyelination is attenuated by sphingosine 1-phosphate signalling. J Cell Sci. 2015;128(21):3878-3887.

51. Sapède D, Dyballa S, Pujades C. Cell lineage analysis reveals three different progenitor pools for neurosensory elements in the otic vesicle. JNeurosci. 2012;32(46):16424-16434.

52. MacPhail RC, Brooks J, Hunter DL, Padnos B, Irons TD, Padilla S. Locomotion in larval zebrafish: Influence of time of day, lighting and ethanol. Neurotoxicology. 2009;30(1):52-58. 